\title{
1 Experimental evidence pointing to rain as a reservoir of tomato phyllosphere microbiota
}

3 Marco E. Mechan-Llontop ${ }^{1,2}$, Long Tian ${ }^{1}$, Parul Sharma ${ }^{1}$, Logan Heflin ${ }^{1}$, Vivian Bernal-

4 Galeano $^{1}$, David C. Haak ${ }^{1}$, Christopher R. Clarke $^{3}$ \& Boris A. Vinatzer ${ }^{1}$

$6 \quad{ }^{1}$ School of Plant and Environmental Sciences, Virginia Tech, Blacksburg, VA 24061, USA.

$7 \quad{ }^{2}$ Current Address: Department of Microbiology and Molecular Genetics and DOE Great Lakes

8 Bioenergy Research Center, Michigan State University, East Lansing, MI 48824, USA.

$9 \quad{ }^{3}$ Genetic Improvement for Fruits and Vegetables Laboratory, Beltsville Agricultural Research

10 Center, U.S. Department of Agriculture-Agricultural Research Service, Beltsville, MD 20705, 11 USA.

13 Corresponding author: B. A. Vinatzer; vinatzer@vt.edu

15 Keywords: bacteriology, genomics, microbiome, plant pathology, rhizosphere and phyllosphere, 16 plants, metagenomics 


\section{ABSTRACT}

20 Plant microbiota play essential roles in plant health and crop productivity. Comparisons of

21 community composition have suggested seeds, soil, and the atmosphere as reservoirs of

22 phyllosphere microbiota. After finding that leaves of tomato (Solanum lycopersicum) plants

23 exposed to rain carried a higher microbial population size than leaves of tomato plants not exposed

24 to rain, we experimentally tested the hypothesis that rain is a so far neglected reservoir of

25 phyllosphere microbiota. Rain microbiota were thus compared with phyllosphere microbiota of

26 tomato plants either treated with concentrated rain microbiota, filter-sterilized rain, or sterile water.

27 Based on 16S rRNA amplicon sequencing, one-hundred and four operational taxonomic units

28 (OTUs) significantly increased in relative abundance after inoculation with concentrated rain

29 microbiota but no OTU significantly increased after treatment with either sterile water or filter-

30 sterilized rain. Some of the genera to which these 104 OTUs belonged were also found at higher

31 relative abundance on tomatoes exposed to rain outdoors than on tomatoes grown protected from

32 rain in a commercial greenhouse. Taken together, these results point to precipitation as a reservoir

33 of phyllosphere microbiota and show the potential of controlled experiments to investigate the role

34 of different reservoirs in the assembly of phyllosphere microbiota. 


\section{INTRODUCTION}

Microbial communities associated with plants, often referred to as plant-associated microbiota and as constituents of the plant microbiome, influence a remarkable number of processes of plant

41 biology and affect plant health and crop yield (Goh et al., 2013, Badri et al., 2013, Hacquard et al.,

42 2015, Lu et al., 2018, Torres-Cortés et al., 2018, Durán et al., 2018, Ritpitakphong et al., 2016,

43 Berg \& Koskella, 2018). The phyllosphere, considered here as the plant compartment that extends

44 from the outside to the inside of the leaf (Morris, 2002, Vacher et al., 2016), harbors a high 45 diversity of microorganisms, with bacteria being the most abundant domain (Lindow \& Brandl, 46 2003, Vorholt, 2012).

Phyllosphere microbiota are exposed to fluctuating environmental stresses, including changes in UV exposure, temperature, water availability, osmotic stress, and humidity (Hirano \& Upper, 2000, Jacobs \& Sundin, 2001, Lindow \& Brandl, 2003, Vacher et al., 2016). The core bacterial phyla that can withstand these environmental stressors include Proteobacteria, Actinobacteria, Bacteroidetes, and Firmicutes (Vorholt, 2012, Bulgarelli et al., 2013). However,

52 at lower taxonomic ranks, the phyllosphere microbiome greatly varies with changing biotic and 53 abiotic factors (Lindemann \& Upper, 1985, Rastogi et al., 2012, Copeland et al., 2015, Wagner et 54 al., 2016).

Culture-independent 16S rRNA amplicon analyses have expanded our knowledge of the composition of the phyllosphere microbiome of several plant species (Knief et al., 2012, Williams 
Stenotrophomonas, Aurantimonas, Thermomonas, Buchnera, Enterococcus, Rubrobacter,

Methylobacterium, Deinococcus, and Acidovorax have all been observed to be associated with the phyllosphere of tomatoes grown in the field (Ottesen et al., 2013, Ottesen et al., 2016, Toju et al.,

63 2019).

Microbes constantly cycle across interconnected habitats maintaining healthy ecosystems (van Bruggen et al., 2019). Importantly for this study, it has been shown that plants represent an important source of microbes that are released as aerosols into the atmosphere (Lindemann et al., 1982, Constantinidou, 1990, Lighthart \& Shaffer, 1995, Šantl-Temkiv et al., 2013, Bowers et al., 2011, Vaïtilingom et al., 2012). The atmosphere then serves as a vehicle for microbial dispersal not only locally, but also globally (Bovallius et al., 1978, Brown \& Hovmøller, 2002, Schmale \& Ross, 2015) and air-borne microbial communities are deposited back to earth surfaces as precipitation. The atmosphere thus represents a crucial route for the dissemination of beneficial and pathogenic species (Polymenakou, 2012, Monteil et al., 2014, Monteil et al., 2016). There is even some evidence that air-borne microbes contribute to the formation of precipitation itself (Christner et al., 2008, Morris et al., 2014, Amato et al., 2015, Amato et al., 2017, Failor et al., 2017). Proteobacteria followed by Bacteroidetes, Actinobacteria, and Firmicutes have been identified as the most common phyla both in the atmosphere (Peter et al., 2014, Hiraoka et al.,

77 2017, Cáliz et al., 2018) as in precipitation (Cáliz et al., 2018, Aho et al., 2020), notable largely

78 the same as the core phyla of the phyllosphere microbiome mentioned earlier.

Microbial community assembly is largely influenced by deterministic (selection) and stochastic (dispersal) processes that determine the complex structure and function of microbiomes

81 (Powell et al., 2015, Zhou \& Ning, 2017, Graham \& Stegen, 2017). This assembly process has

82 been extensively studied in roots, which recruit microbial communities from the surrounding soil 
83 (Fitzpatrick et al., 2018, Pérez-Jaramillo et al., 2019). However, sources that drive the phyllosphere microbiome assembly are still under debate. It has been suggested that soil is the major reservoir of the phyllosphere microbiome (Zarraonaindia et al., 2015, Wagner et al., 2016, Grady et al., 2019). In fact, it has been observed that the leaf microbiome reflects soil bacterial diversity at an early stage of growth but significantly differs as plants grow and mature (Copeland et al., 2015).

88 On the contrary, (Maignien et al., 2014, Ottesen et al., 2016) found evidence that dry deposition of air-borne microbes constitutes an important source of phyllosphere microbiota.

A few studies have also explored wet deposition of air-borne microbes in precipitation as 91 a potential reservoir of phyllosphere microbiota. For example, (Morris et al., 2008) concluded that

92 the plant pathogen Pseudomonas syringae is disseminated through the water cycle because of its 93 ubiquitous presence in compartments of the water cycle and plants. Using whole genome 94 sequencing, (Monteil et al., 2016) confirmed these conclusions by finding that $P$. syringae bacteria

95 isolated from diseased cantaloupe plants and $P$. syringae isolates from rain, snow, and irrigation 96 water were members of the same population. Recently, rain was also found to affect the overall 97 composition of plant phyllosphere microbiota but it was not determined if rain-borne bacteria were 98 the source of the observed shifts (Allard et al., 2020). Moreover, it is well known that fungal spores 99 are released from plants, travel long distances through the atmosphere, and can be deposited back 100 on plants with rain (Woo et al., 2018).

102 bacterial population size than the phyllosphere of tomato plants not exposed to rain, we 103 hypothesized that rain-borne bacteria might contribute to the assembly of phyllosphere microbiota 104 beyond pathogenic bacteria and fungi. Putative rain-borne tomato phyllosphere colonizers were 
105 identified in a series of controlled laboratory experiments and by comparing the composition of tomato phyllosphere microbiota of plants naturally exposed to rain with those not exposed to rain.

\section{MATERIALS AND METHODS}

111 tomato seeds of the cultivar 'Rio Grande' were germinated in autoclaved soil (60 min/ fast cycle).

112 For growth under laboratory conditions, plants were kept for 4 weeks on shelves under $14 \mathrm{~h}$ of

113 light and $10 \mathrm{~h}$ of dark. For exposure to outdoor conditions, 4-week-old tomato plants were

114 transplanted into large plastic pots. Pots were then either transported to a research farm and placed

115 on gravel near a maintained lawn (Kentland Farm, Blacksburg, VA, USA) or put on the flat roof

116 of the 3-story Latham Hall research building at Virginia Tech (Blacksburg, VA, USA) several

117 meters above and away from soil and vegetation.

118 Culture-dependent analysis of phyllosphere microbiota. Leaf disks were aseptically

119 collected with a \#1 cork borer and placed in a tube containing $200 \mu \mathrm{L}$ of sterile $10 \mathrm{mM} \mathrm{MgSO}_{4}$

120 solution and 3 glass beads. Tubes were placed in a mini bead beater (Biospec Products, Inc.,

121 Bartlesville, OK, USA) and shaken for $2 \mathrm{~min}$ to release bacterial cells. Serial dilutions were plated

122 on R2A plates supplemented with cycloheximide to inhibit fungal growth. Plates were incubated

123 at room temperature and colony-forming units were counted 4 days later.

124 Rain collection. Rain was collected as described in (Failor et al., 2017). In short, 125 autoclavable bags were wrapped in aluminum foil and autoclaved for $40 \mathrm{~min} /$ fast cycle. Trash cans 126 were arranged away from structures on the roof of the Latham Hall research building. Surfaces of 127 containers were sprayed with $75 \%$ ethanol to prevent contamination. Sterile bags were placed in 
128 the cans and the lid placed back on top until the beginning of a rainfall event, at which point they

129 were removed. The lids of three cans were then removed but one can was kept closed during the

130 precipitation event as a negative control. After the end of precipitation events, 1 liter (L) of sterile

131 water was poured into the negative control can, simulating the precipitation event. After rainfall

132 events ended, bags containing rain water were removed and placed at $4^{\circ} \mathrm{C}$ until processing.

133 For DNA extraction, three L of rainwater were vacuum filtrated (reusable filter holders

134 from Thermo Scientific Nalgene, USA) through a $0.2 \mu \mathrm{m}$ pore filter membrane (Supor ${ }^{\circledR} 200$ PES

135 membrane Disc Filter, PALL, USA). Filters were removed using sterile tweezers, placed into a 15

$136 \mathrm{~mL}$ Eppendorf tube and stored at $-80^{\circ} \mathrm{C}$ until processing.

137 Rain as bacterial inoculum and plant treatments. Tomato plants were grown in the

138 laboratory for four weeks as described above for the culture-dependent analysis of phyllosphere

139 microbiota. Two L of rainwater were vacuum-filtered as described under rain collection. To

140 concentrate the bacterial microbiota present in rain hundred fold, the filter membranes were

141 incubated for 10 min with shaking in $20 \mathrm{~mL}$ of sterile water, which was then used as inoculum

142 (referred to as concentrated rain microbiota or 100X-rain from now on). The rain that passed

143 through the filter was used as bacterial-free inoculum (referred to as filtered rain or filter-sterilized

144 rain). Autoclaved double-distilled water was used as sterile water treatment. Groups of four plants

145 placed together into 13 inch x 16 inch plastic bags were sprayed until run off. The bags were left

146 open for two hours to let the plants dry before the day 0 time point DNA extraction from two of

147 the four tomato plants. Bags were then closed for 2 days to create a high humidity environment

148 favorable to plant colonization after which they were kept open for 5 days until the day 7 time

149 point DNA extraction from the remaining two tomato plants. 
Growth of outdoor and greenhouse tomatoes. Tomato plants were purchased at Home

151 Depot at the beginning of June 2019 and transplanted into pots and placed on the flat roof of the

152 Latham Hall building on the Virginia tech campus by setting them on a large, clean sheet of plastic

153 to reduce contamination from the roof floor. Plants were regularly waters without touching leaves.

154 One set of plants were brought indoors before each rainfall event while the other set of plants were

155 kept outside to be exposed to rainfall. Each rainfall event was collected and DNA extracted as

156 described above and leaves from both sets of plants were harvested and DNA extracted as

157 described below.

Harvesting plants leaves. All plant leaves were removed and collected in a ziplock plastic

159 bag. Sterile distilled water was added $(300 \mathrm{~mL})$ and samples were sonicated for 10 minutes using

160 a 1510 BRANSON sonicator (Brandsonic, Mexico) (Ottesen et al., 2013). The leaf wash was

161 vacuum-filtered on to the same kind of $0.22 \mu \mathrm{m}$ pore filter described above to collect microbial

162 cells dislodged from leaf tissue during sonication, as described above. Filter membranes were

163 placed into a $15 \mathrm{~mL}$ Eppendorf tube and stored at $-80^{\circ} \mathrm{C}$ until processing.

164 Plant leaves were also collected using the same procedure inside a commercial greenhouse

165 where tomatoes were grown either hydroponically or in non-sterilized soil under an organic

166 production regiment.

167 DNA extraction. DNA extraction from all the $0.22 \mu \mathrm{m}$ filter membranes was performed

168 using the Power Water DNA isolation kit (Qiagen, USA) according to the manufacturer's protocol

169 with minor modifications. DNA concentration and quality were assessed by UV

170 spectrophotometry (NanoDrop 1000, Thermo, USA) and visualized on a $1 \%$ agarose gel.

171 Library preparation and sequencing. For the 16S rRNA amplicon sequencing we used

172 the barcoded primers a799wF (5'AMCVGGATTAGATACCCBG3') and new1193R 
173 (5'ACGTCATCCCCACCTTCC3'). A 28 cycle PCR was performed using the HotStarTaq Plus

174 Master Mix Kit (Qiagen, USA) under the following conditions: $94^{\circ} \mathrm{C}$ for 3 minutes, followed by

17528 cycles of $94^{\circ} \mathrm{C}$ for 30 seconds, $53^{\circ} \mathrm{C}$ for 40 seconds and $72^{\circ} \mathrm{C}$ for 1 minute, after which a final

176 elongation step at $72^{\circ} \mathrm{C}$ for 5 minutes was performed. After amplification, PCR products obtained

177 from the various samples were mixed in equal concentrations and purified using Agencourt

178 Ampure beads (Agencourt Bioscience Corporation, Beverly, MA, USA). All steps from PCR to

179 paired-end $(2 \times 300 \mathrm{bp})$ amplicon sequencing on the Illumina MiSeq platform were performed at

180 Molecular Research LP (MR DNA ${ }^{\mathrm{TM}}$, Shallowater, TX, USA).

For metagenomic sequencing using the Illumina platform, total DNA was sequenced using

$182150 \mathrm{bp}$ paired-end reads on the HiSeq 4000 Illumina platform, Duke University Sequencing and

183 Genomic Technologies Shared Resource, Durham, NC, USA. For metagenomic sequencing using

184 the Nanopore platform, DNA libraries were prepared following the '1D Native barcoding genomic

185 DNA protocols (SQK-LSK109 and EXP-NBD104) provided by Oxford Nanopore Technologies

186 (ONT).

187 Bioinformatic analysis. Raw $16 \mathrm{~S}$ rRNA paired-end sequences were processed by

188 Molecular Research LP (MR DNA ${ }^{\mathrm{TM}}$, Shallowater, TX, USA) as follows: 1) reads were joined

189 together after q25 trimming of the ends and reoriented in the 5'-3' direction, 2) barcodes and

190 primer sequences were removed, and 3) sequences shorter than 200bp, sequences with ambiguous

191 base calls, and sequences with homopolymer runs exceeding 6 bp were removed. Operational

192 taxonomic units (OTU) were assigned using the open source Quantitative Insights into Microbial

193 Ecology (QIIME) version 1.9.1bioinformatic pipeline (Caporaso et al., 2010), using the open-

194 reference protocol at 97\% sequence identity with UCLUST as the clustering tool and SILVA 
195 release 128 (Quast et al., 2013) as the database. All OTUs annotated as mitochondria, chloroplasts, 196 cyanobacteria, unassigned, and OTUs with fewer than five reads were removed from the dataset. The QIIME-generated output file in the Biological Observation Matrix (BIOM) format was 198 used for downstream data analysis and visualization in R version 3.3 using the Vegan (Oksanen 199 J., 2020), Phyloseq 1.26.1 and ggplot2 3.3.2 packages (McMurdie \& Holmes, 2013, Hadley, 2009). 200 Samples were rarefied to the lowest sample depth to compute diversity analysis. The rain 201 core microbiome analysis was performed with the microbiome R package (Lahti L., 2020) using 202 a detection threshold of $0.1 \%$ and $100 \%$ as a prevalence threshold. Alpha diversity was assessed 203 using observed OTUs, Shannon, and Simpson indices. Differences in alpha diversity were 204 determined by pairwise Wilcoxon rank sum test with the Holm correction method. Beta diversity 205 was analyzed based on unweighted UniFrac distance, weighted UniFrac distance, and Bray-Curtis 206 dissimilarity. Differences in beta diversity were determined using PERMANOVA as implemented 207 in adonis2 (from vegan 2.5-6 using models with 999 permutations, adonis2(dist.matrix $208 \sim$ Treatment*TimePoint + DateExperiment, permutations $=999)$. Dissimilarity matrices were 209 visualized using the Principal Coordinates Analysis (PCoA) ordination method as implemented in 210 the Phyloseq package. DESeq2 (Love et al., 2014) was used to identify OTUs that were 211 differentially abundant across treatment groups and time points. OTUs were filtered using a False 212 Discovery Rate (FDR) cutoff of 0.01.

213 For metagenomic data analysis, raw 150 bp paired-end reads from Illumina were processed 214 to remove short and low quality reads using Trimmomatic (version 0.38) (Bolger et al., 2014). 215 Reads with an average per base quality below 30 and read length below $150 \mathrm{bp}$ were filtered out. 216 Filtered reads were then classified taxonomically using Centrifuge (version 1.0.4) (Kim et al., 
217 2016) and Sourmash version 2.0.0 (Brown, 2016) only retaining species that were identified by

218 both classifiers.

219 For Oxford Nanopore Technologies sequencing, the fast5 files containing the raw reads

220 obtained from the MinION sequencer were base-called using Guppy (v3.3.2). The ONT workflow

221 What is in my pot (WIMP v2019.7.9) was used for bacterial identification and a classification

222 (Juul et al., 2015).

224 RESULTS

tomato plants not exposed to rain. Our investigation into the role of rain in shaping the

233 the same period (Figure 1A). Interestingly, even plants grown on the roof of a campus research

234 building, which minimized microbial dispersal from soil and plants compared to the farm

235 environment, had bacterial populations that were significantly larger compared to those of plants

236 grown indoors (Figure 1B). These results suggest that air-borne and rain-borne bacteria through

237 dry and/or wet deposition had colonized the tomato plants grown outside.

To test the effect of rain on the bacterial population size in the tomato phyllosphere under

239 controlled conditions, we collected rain and used it as an inoculum to treat 4-week old tomato 
240 plants that had been grown under laboratory conditions. Seven days after plants were treated with

241 rain, they carried a significantly larger bacterial population count compared to plants that had been

242 treated with autoclaved rain (Figure 1C). This result suggested that at least some rain-borne

243 bacteria are able to colonize plant leaves efficiently and may thus impact bacterial population

244 composition in the phyllosphere.

245 Rain-borne microbiota in Blacksburg, Virginia, are highly variable. As a first step

246 towards identifying which bacterial taxa present in rainfall may efficiently colonize the tomato

247 phyllosphere, we characterized the bacterial diversity associated with nine rainfall events during

2482015 and 2016. Rainfall was collected in sterile plastic bags on the same roof of the research

249 building previously used to grow tomatoes, DNA was extracted, and the 16S rRNA gene was

250 amplified and sequenced (Table 1). In total, 1,186,365 short reads were obtained. After 97\% OTU

251 clustering and removal of all non-bacterial and unassigned reads, a total of 892,142 reads

252 remained. All samples were rarefied to 6419 reads per sample and 5958 OTUs overall were

253 identified. Rarefaction curves (Supplementary Figure 1) show that this is an underestimate of the

254 total number of OTUs since not all samples were sequenced to saturation. The number of OTUs

255 per rarefied sample ranged from 541 (June 2019) to 1782 (April 2016).

Taxonomic diversity analysis revealed Proteobacteria, Actinobacteria, Bacteroidetes,

257 Acidobacteria, and Firmicutes to be the dominant taxa at the phylum level. At the class level,

258 Alphaproteobacteria followed by Gammaproteobacteria, Actinobacteria, Acidobacteria, and

259 Bacilli were most abundant. However, there were considerable differences among samples. For

260 example, Chlamydiae represented the most abundant taxon in the rain sample collected in August

2612015 at $36 \%$ relative abundance, represented only $0.4 \%$ in the sample collected in July 2016 , and

262 were less than $0.1 \%$ in all other samples. Deltaproteobacteria were only present in August 2015, 
263 May 2016 and July 2016 at 12.79\%, 1.42\%, and 17.03\% relative abundance, respectively.

264 Actinobacteria were most abundant in rain samples collected in April $2016(25.56 \%)$ and

265 December $2016(16.29 \%)$ while they only represented $2.18 \%$ of the October 2016 sample.

266 Gammaproteobacteria represented $80 \%$ of relative abundance in June 2019 but only averaged

$26728.41 \%$ in the other samples (Figure 2A).

268 Even more dramatic differences in relative abundance between samples were observed at

269 the genus level (Figure 2B). However, seventeen OTUs were identified across all nine samples

270 when setting a detection threshold of $0.1 \%$. These OTUs belong to the following genera (listed in

271 order of decreasing relative abundance): Acidiphilium, Bryocella, Beijerinckiaceae 1174-901-12,

272 Methylobacterium, Massilia, Burkholderiaceae DQ787673.1.1527, Pantoea, Pseudomonas and

273 Sphingomonas (Supplementary Figure 2).

275 phyllosphere of lab-grown tomato plants. Next, we decided to determine if inoculation with rain

276 microbiota would not only increase the size of the tomato phyllosphere microbiota as seen in

277 Figure 1 but also change its composition because of colonization of tomato leaves by rain-borne

278 bacteria. In six independent experiments, 100-fold concentrated rain microbiota (100X-rain)

279 derived from six out of the nine collected rainfall events described above (April 2015, August

280 2015, March 2016, April 2016, May 2016, July 2016) were used to inoculate tomato plants. In

281 parallel, a separate set of tomato plants were treated each time with filter-sterilized rain obtained

282 from the concentration step above. For each of the 2016 experiments, additional tomato plants

283 were treated with sterile water. 16S rRNA amplicons were prepared and sequenced from DNA

284 extracted from leaf washes of tomato plants two hours after treatments (day-0) and seven days

285 later (day-7). For the March 2016 and May 2016 experiments enough rain and enough plants were 
available so that each treatment was done in duplicate. For the April 2016 experiment, two day-7

287 100X-rain samples were taken. For the other three experiments, only one sample per treatment and

288 time point was processed.

In total, 3,291,016 reads were obtained from 45 phyllosphere samples (Supplementary

Table 1). After $97 \%$ OTU clustering and removing all non-bacterial and unassigned reads, a total of $3,118,320$ reads remained in the data set. Rarefaction curves show that most of the samples were deeply sequenced (Supplementary Figure 1).

After subsampling to 6,670 reads per sample, we identified a total of 9,923 OTUs and measured the alpha diversity based on the total number of observed species and by Shannon and plants at day 0 and day 7 . Although alpha diversity of rain microbiota was highly variable, the number of observed OTUs in rain was significantly higher than day-7 samples for all three treatments ( $\mathrm{p}$-values of $0.020,0.050$, and 0.028 respectively as determined by the pairwise number of observed OTUs significantly decreased in plants treated with 100X-rain from day 0 to filtered-rain ( $\mathrm{p}$-value 0.023). No significant differences in alpha diversity were observed between 304 day 0 and day 7 for plants treated with sterile-water. 
309 from unweighted Unifrac distances and Bray-Curtis dissimilarity separated phyllosphere samples

310 along both axes. Therefore, bacterial communities on lab-grown tomato plants were even more

311 dissimilar from each other than the dissimilarity between rain communities. A permutational

312 multivariate analysis of variance (PERMANOVA) for Bray-Curtis dissimilarity revealed that date

313 of experiment, day of sampling (day 0 versus day 7), and treatment (100X-rain, filter-sterilized

314 rain, and sterile water) were all significantly associated with bacterial community composition.

315 (Supplementary Table 3). In regard to the date of experiment, it can be seen in panels 4B and 4C

316 how samples clearly cluster by date of experiment along the $\mathrm{X}$ axis. Since this clustering is

317 independent of treatment and day of sampling, the starting tomato phyllosphere population appears

318 to have been different between experiment dates. Secondly, day 7 samples differed from day 0

319 samples on the $\mathrm{Y}$ axis independently of which treatment was applied revealing that inoculation

320 and incubation under high humidity of plants by itself shifted the composition of the tomato

321 phyllosphere population. Thirdly, although treatment was significant, there was interaction

322 between treatment and date of experiment. Therefore, the effect of 100X-rain was not significantly

323 different compared to the effect of filter-sterilized rain or sterile water. In other words, beta

324 diversity analysis was unable to reveal if rain-borne bacteria present in the 100X-rain treatments

325 affected the tomato phyllosphere community, possibly because of the differences in the

326 composition of the microbial rain and phyllosphere communities between experiments.

After finding that beta diversity analysis was inconclusive, we decided to compare the

328 actual taxonomic diversity between samples. We observed an enrichment in Proteobacteria in the

329 tomato phyllosphere on day 7 compared to the day 0 regardless of treatment. In contrast, relative

330 abundance of Actinobacteria and Firmicutes was dramatically reduced 7 days post-treatment in all

331 plants treated with 100X-rain but not after treatment with filter-sterilized rain or sterile water 
332 (Figure 5). At the class level, Gammaproteobacteria were significantly enriched in all tomato

333 phyllosphere day 7 samples. Actinobacteria and Bacilli were reduced on day 7 in plants treated

334 with 100X rain compared with filter-sterilized rain or sterile water. Unexpectedly, no consistent

335 increase of any taxon at either phylum, class, or genus level was observed from day 0 to day 7 for

336 plants treated with 100X-rain alone.

One-hundred and four rain-borne OTUs increased significantly in relative

microbiota. Since we did not find any taxon between genus and phylum level that exclusively increased from day 0 to day 7 on tomato plants treated with $100 \mathrm{X}$-rain, we wanted to determine if we could find any individual rain-borne OTUs that did so. To do this we used DESeq2 (Love et al., 2014) as it is relatively robust to small and unequal sample sizes as in the present study.. rain and found that one-hundred and four OTUs (out of a total of 7,994 OTUs) significantly increased (Supplementary Table 4). These OTUs belonged to the genera Massilia (27 OTUs), Pantoea (18 OTUs), Duganella (13 OTUs), Pseudomonas (11 OTUs), Enterobacter (5 OTUs),

347 Flavobacterium (3 OTUs), Janthinobacterium (2 OTUs), and Curtobacterium (1 OTUs). 16

348 unknown OTUs from the Burkholderiaceae and 5 unknown OTUs from the Enterobacteriaceae

349 families significantly increased in relative abundance as well (Figure 6A). Importantly, not a 350 single OTU significantly differed in abundance between day 0 and day 7 on tomato plants treated 351 with either filtered-rain or dd-water. This suggests that the OTUs which increased in relative 352 abundance from day 0 to day 7 on tomatoes after treatment with 100X-rain originated from rain 353 and were not members of the bacterial community present in the tomato phyllosphere prior to 354 inoculation. 
We then complemented the previous comparison with a slightly different analysis comparing the bacterial composition of rain microbiota with phyllosphere microbiota seven days after treatment with 100X-rain. We observed that thirty-five rain-borne OTUs out of a total of 5,958 OTUs had a significantly higher relative abundance on tomatoes at day-7 compared to their relative abundance in rain (Figure 6B). These OTUs (in order of decreasing differential

360 abundance) mostly belonged to the same genera as the genera identified in the day-0 to day-7

361 comparison : Massilia, Pseudomonas, Pandoraea, Streptomyces, and Pantoea. The genus Pantoea

362 was the genus that most consistently increased in relative abundance. In fact, Pantoea was detected

363 in all rain collections (Figure 7A) and reached a high relative abundance (between 4\% - 44\%) in

364 the tomato phyllosphere after 7 days each time its abundance in rain was above 1\% (observed 4 365 out of 6 times). Genus-level abundance in rain and phyllosphere samples are also shown for Flavobacterium, Janthinobacterium, Pseudomonas, Methylobacterium, and Massilia, which all

367 successfully colonized the tomato phyllosphere each time they were detected in rain (Figure 7BE).

In contrast, 61 OTUs found in rain samples significantly decreased in relative abundance 370 by day 7 suggesting that these rain-borne taxa were definitely not able to colonize tomato leaves.

371 These OTUs belonged to the following genera (list of the first 10 bacterial genera in order of 372 decreasing differential abundance): Acidiphilium, Beijerinckiaceae-1174-901-12, Bryocella, 373 Actinomycetospora, Methylobacterium, Methylocella, Granulicella, Belnapia, Modestobacter,

374 and Blastococcus. The genus Acidiphilium best exemplifies this group of taxa. It was detected at 375 high relative abundance in most rain samples, it was observed on plants treated with 100X-rain on 376 day 0, but it was never found on plants treated with 100X-rain on day 7 (Figure 7G). This clearly 
377 shows that Acidiphilium is a common rain-borne bacterial genus that does not include any

378 members able to colonize the tomato phyllosphere.

Several other pairwise comparisons were made to gain additional insights into differences

380 in composition between microbiota at the OTU level. For example, we determined which OTUs

381 were present in significantly higher abundance in rain compared to tomatoes treated with sterile

382 water on day 0 to identify OTUs that are commonly present in rain but present in low abundance

383 (or not at all) on laboratory-grown tomato plants (Supplementary Figure 3A). One hundred

384 seventy-four such OTUs (out of a total of 5,958 OTUs present in rain) were identified. Most of

385 these OTUs belonged to the following genera (list of the first 10 bacterial genera in order of 386 decreasing differential abundance in rain): Caedibacter, Tumebacillus, Acidiphilium, 387 Methylobacterium, Rhodovastum, Belnapia, Sphingomonas, Methylocella, Bacillus and Pantoea. grown tomatoes (tomatoes at day 0 after being treated with sterile water) than in rain

390 (Supplementary Figure 3A). These taxa are thus common inhabitants of tomatoes grown under 391 our laboratory conditions in the absence of rain. They mostly belonged to the following genera 392 (list of the first 10 bacterial genera in order of decreasing differential abundance):

393 Hyphomicrobium, Rhodanobacter, Chryseobacterium, Burkholderia, Paenibacillus,

394 Nocardioides, Pandoraea, Bacillus, Novosphingobium, and Streptomyces.

396 OTUs were observed at high relative abundance in rain samples as well as in the tomato 397 phyllosphere at day 0 independent of treatment and on day 7 after sterile-rain and dd-water 398 treatments but not after 100X-rain treatments (Figure 7H). This suggests that Bacillus OTUs were 
present in rain as well as on lab-grown tomatoes but were outcompeted by other rain-borne bacteria added with the 100X-rain treatments.

To more precisely identify the OTUs that represented the most efficient tomato colonizers, we used metagenome shotgun sequencing to re-sequence the microbiota associated with rain sequencing approach generated 260,035,170 short-reads. After quality control, 7,543,305 reads

411 Cedecea neteri, and an unnamed Massilia species. Additional Pantoea, Massilia, Pseudomonas,

412 Janthinobacterium, and Enterobacter species ranked highly as well. The metagenomic analysis

413 thus mostly confirmed and refined our 16S rRNA results of which rain-borne taxa are the most 414 effective colonizers of the tomato phyllosphere.

417 present in rain that efficiently colonized tomato leaves under laboratory conditions, we tested the

418 hypothesis that these taxa would be abundant in tomato plants grown outdoors in pots containing 419 autoclaved soil on the roof of the same research building where we had collected rain samples 420 earlier (7 samples) but be missing, or at least be underrepresented, in phyllosphere microbiota of 421 greenhouse-grown tomatoes that had never been exposed to rain. This second set of plants included 
422 tomato plants grown in a hydroponic system (29 samples) and tomato plants grown in soil (18

423 samples), both in a commercial greenhouse (Supplementary Table 5). In total, we obtained

$4244,166,519$ reads. After 97\% OTU clustering and removing all non-bacterial and unassigned reads,

425 a total of 3,080,204 reads remained. Samples were rarefied to 2,546 reads per sample and 10,525

426 OTUs overall were identified. Taxonomic diversity analysis revealed Proteobacteria, Firmicutes,

427 Actinobacteria and Bacteroidetes to be the dominant bacterial taxa (Figure 8A), the same phyla

428 identified on the lab-grown tomatoes. Alpha diversity analysis supported by the total number of

429 observed species, and by the Shannon and Simpson diversity indices (Figure 8B) together with a

430 pairwise comparisons using the Wilcoxon rank sum test showed significant differences in the

431 number of OTUs between the hydroponic and the soil system (Supplementary Table 6).

432 To identify any OTUs present at significantly higher relative abundance in tomato plants

433 grown outside exposed to rain compared to the tomato plants grown in the greenhouse

434 hydroponically in the absence of rain, DESeq2 was used again. Forty OTUs from the genera

435 Pandoraea, Curtobacterium, Massilia, Gemmatirosa, Kineococcus, Methylobacterium,

436 Sphingomonas, Buchnera, Alloiococcus, Pseudomonas, and Streptococcus were found (Figure

437 9A). Similarly, 58 OTUs from the genera Pantoea, Pelomonas, Kineococcus, Methylobacterium,

438 Massilia, Aureimonas, Buchnera, Alloiococcus, Pseudomonas, Streptococcus, and Sphingomonas

439 were of significantly higher relative abundance on the tomato plants grown outside exposed to rain

440 compared to the tomato plants growing in soil never exposed to rain in the greenhouse (Figure

441 9B). Note that, as we hypothesized, OTUs in the genera Massilia, Curtobacterium, Pseudomonas,

442 and Pantoea, were among the same genera as the OTUs identified to significantly increase in

443 relative abundance in the phyllosphere of tomato plants treated with concentrated rain microbiota

4447 days post inoculation. However, unexpectedly, the actual OTUs of these genera identified in this 
445 comparison were not the same as those identified in the controlled laboratory experiments (see 446 discussion section for possible explanations).

Finally, we performed another small experiment collecting rain on the same roof as in the

448 previous experiments and exposing one set of tomato plants to all rain events while taking another

449 set of plants inside before each rain event. From one rain event (June 10, 2019), phyllosphere

450 communities from a single tomato plant exposed to rain (collected on June 21, 2019), and another

451 tomato plant not exposed to rain (collected on the same date) were sequenced together with a

452 sample of the corresponding rainfall using ONT' MinION platform. A total of 194,591 long reads

453 were analyzed using the ONT taxonomic classifier WIMP. Table 3 shows the most abundant

454 species present in the three samples ranked by relative abundance on the tomato plant exposed to

455 rain. Yet again, species of the genera Massilia, Pantoea, Methylobacterium, Pseudomonas, and

456 Janthinobacterium (and some additional species outside of these genera) were present in the rain

457 sample as well as in the tomato sample exposed to rain but absent (or almost absent) in the tomato

458 sample not exposed to rain. While this experiment was too small for any statistical analysis, the

459 result is consistent with the laboratory results as well as the comparison of tomatoes grown outside

460 exposed to rain with those grown in a greenhouse not exposed to rain.

\section{DISCUSSION}

464 While our general understanding of the plant microbiome has increased dramatically over the last

465 few years, the basic question of where the bacteria that colonize and inhabit the phyllosphere

466 originate from has remained unanswered. The main approach in trying to answer this question has

467 been to make comparisons of the composition of the phyllosphere microbiome with the 
468

469

470

471

472

473

474

475

476

477

478

479

480

481

482

483

484

485

486 487 in relative abundance on tomato plants treated with concentrated rain microbiota (100X-rain) but 488

microbiomes of putative reservoirs (Maignien et al., 2014, Zarraonaindia et al., 2015, Ottesen et al., 2016, Wagner et al., 2016, Grady et al., 2019). To complement this approach, here we used controlled laboratory experiments.

We decided to focus on rain since previous studies provided evidence that at least the bacterial leaf pathogen, Pseudomonas syringae, may be disseminated by precipitation and efficiently colonize the plant phyllosphere (Monteil et al., 2016, Morris et al., 2008). Moreover, at least temporary shifts in the composition of phyllosphere communities after rain events were observed (Allard et al., 2020). In a first step, we found that tomato plants naturally exposed to rain outdoors, or simply sprayed with rainfall when grown in the lab, carried significantly higher bacterial populations sizes compared to lab-grown plants that had not been treated with rainfall or that were treated with sterilized rainfall. While plants grown outside may have also been colonized by airborne bacteria through dry-deposition or by soil-borne bacteria after being splashed during rainfall events, the observation that treating lab-grown plants with rain caused the bacterial population size to increase more than spraying lab-grown plants with sterilized rain was a strong indication that the observed increase in population size was due to the colonization and growth of rain-borne bacteria.

To dig deeper into the possibility that rain harbors bacteria that can effectively colonize and grow on tomato leaves, we decided to characterize the taxonomic composition of rainfall events and then determine if any of the identified members of the rain microbiota would increase not on tomato plants treated with filter-sterilized rain or sterile water. We decided on using concentrated rain microbiota instead of rain since we had previously found that rain contained as few as $4 \times 10^{3} \mathrm{CFU}^{-1}$ (Failor et al., 2017) and the maximum volume that we can spray on a tomato 
491 plant before water runs off the leaves is only $10 \mathrm{~mL}$. Therefore, as few as 40 bacteria may get

492 inoculated on an entire plant using rain as inoculum in a lab experiment, which we deemed not to

493 be enough to lead to a bacterial population size that could be analyzed using 16S rRNA amplicon

494 sequencing. We thus used 100X-rain concentrate, acknowledging that we artificially aided rain-

495 borne bacteria in our experiment.

496 The most important observation from the characterization of the rainfall microbiota was

497 that each collected rainfall event harbored a very different bacterial community. This is in line with

498 other recent results on rainfall collected in the USA, Europe, and Asia (Aho et al., 2020, Cáliz et

499 al., 2018, Woo \& Yamamoto, 2020) that showed that the taxonomic composition of microbiota in

500 rain changes with origin of air masses and season. This meant for our experiment that we could

501 not expect to find the same taxa to colonize and grow on tomatoes in each inoculation experiment.

502 It was also important to use appropriate controls when inoculating lab-grown tomato plants

503 with 100X-rain. Importantly, tomato plants were not grown in sterile conditions. Therefore, they

504 already carried microbiota at the time of inoculation and simply spraying these plants with water

505 and incubating them at high humidity (as we did to favor plant colonization) could be expected to

506 lead to changes in relative abundance of the pre-existing microbiota. Moreover, rain contains

507 nutrients and bacteriophages that are not removed by filtering and that will also affect pre-existing

508 microbiota. Therefore, we used both, filter-sterilized rain (including nutrients and possibly

509 bacteriophages but no bacteria) and autoclaved double-distilled water (expected to contain neither

510 nutrients nor bacteriophages nor bacteria) as controls.

511 Comparison of rain microbiota with phyllosphere microbiota on day-0 and day-7 for the

512 three different treatments yielded some results confirming our hypothesis that rain contains

513 effective colonizers of tomato leaves while other results were ambiguous. Firstly, the observed 
514 drop in alpha diversity for phyllosphere microbiota from day-0 to day-7 after treatment with 100X-

515 rain is in agreement with the expected increase in relative abundance of some effective tomato leaf

516 colonizers accompanied by a decrease in relative abundance of many rain-borne bacteria that are

517 not adapted to tomato leaves and that are thus not effective tomato colonizers. The observed

518 decrease in alpha diversity from day-0 to day-7 for the sterile rain treatment may have been due to

519 growth of some pre-existing tomato phyllosphere members that thrived under the high humidity

520 conditions applied for two days after inoculation and the nutrients added with the rain water, which

521 helped them outcompete other members of the pre-existing microbiota. It is also possible that

522 bacteriophages present in the filter-sterilized rain reduced the relative abundance of some

523 phyllosphere members to below the detection limit.

524 In regard to beta diversity, we had expected the composition of the day 0 microbiota to be

525 similar to each other because tomato plants of the same cultivar were grown in autoclaved soil in

526 relatively stable laboratory conditions. Therefore, it was surprising to find phyllosphere samples

527 to differ more from each other (even on day-0 after sterile water treatments) compared to the

528 differences among rain samples. This was the case for weighted UniFrac distances, unweighted

529 UniFrac distances, and the Bray-Curtis dissimilarity static. A possible explanation is that the

530 composition of the phyllosphere microbiota of our lab-grown tomatoes was determined by

531 stochastic processes because of the low concentration of plant-associated bacteria present in the

532 indoor air and the autoclaved soil that was used for growing.

533 Another unexpected result was that beta diversity significantly changed from day-0 to day-

5347 for all three treatments. Although the high humidity maintained for two days after inoculation

535 was expected to have some effect on the phyllosphere community, we still expected 100X-rain to

536 have a stronger effect on beta diversity than sterile water. Similarly unexpected, the taxonomic 
composition at the phylum, class, and genus level on day-0 and day-7 did not reveal any consistent change unique to the treatment with 100X-rain compared to filter-sterilized rain or sterile water.

Taken together, these results suggested that, if they existed, tomato leaf colonizers present in rain were individual species and changes in abundance of these individual species were not evident from the overall comparison of beta diversity or taxonomic composition at higher taxonomic ranks.

in RNA-seq experiments, which have similar challenges to OTU tables, and which has been shown to be effective for smaller OTU datasets like ours (Weiss et al., 2017). We made several comparisons. Most importantly, not a single OTU significantly increased from day 0 to day 7 after treatment with filter-sterilized rain or sterile water but 104 OTUs increased significantly after after treatment with filter-sterilized rain or sterile water, they were most likely rain-borne.

551 analysis identified 18 OTUs of this genus that significantly increased in relative abundance from 552 day 0 to day 7 after being treated with 100X-rain. Importantly, Pantoea OTUs were also present 553 in all rain samples (including the one sequenced with ONT's MinION). Moreover, the species $P$. 554 agglomerans and $P$. vagans were identified as the most abundant species in several of the tomato 555 phyllosphere day 7 samples after being treated with 100X-rain based on metagenomic sequencing.

556 Also, two Pantoea OTUs were present in significantly higher relative abundance in tomatoes 557 grown outside compared to tomato grown organically inside a commercial greenhouse, and $P$. 558 agglomerans and $P$. vagans were both identified in the rain metagenome as well as in the 559 metagenome of a tomato plant exposed to rain but not in a tomato plant not exposed to rain using 
ONT MinION sequencing. Moreover, the genus Pantoea is well known to include plant pathogenic

561 bacteria and beneficial plant-associated bacteria (Coutnho \& Venter, 2009, Walterson \&

562 Stavrinides, 2015, Mechan Llontop et al., 2020), we previously identified 192 Pantoea isolates in

563 a culture-dependent study of precipitation samples (Failor et al., 2017), and Pantoea species were

564 recently identified both in rainfall before and after falling through a forest canopy, with higher

565 relative abundance in the throughfall samples (Ladin et al., 2021). Therefore, based on the results

566 obtained here and data in previous literature, members of the genus Pantoea are likely

567 phyllosphere inhabitants that originate from rainfall.

Another rain-borne genus that includes species that appear to successfully colonize tomato plants is Massilia. Members of this genus were found in all rain samples (those analyzed by $16 \mathrm{~S}$ rRNA amplicon sequencing and the one sequenced with ONT's Minion). Twenty-seven OTUs of this genus significantly increased between day- 0 phyllosphere samples and day-7 samples for

572 100X-rain treated plants. Two Massilia species were also found among the species with the highest 573 relative abundance in the metagenomic sequences of the 100X-treated tomato samples on day 7.

574 One Massilia OTU each was more abundant in tomatoes grown outdoors compared to 575 hydroponically or organically grown tomatoes in the commercial greenhouse, respectively. Four 576 Massilia species were found in the rain sample and tomato sample grown outdoors but not in the 577 tomato plant not exposed to rain when using ONT MinION sequencing. As with Pantoea, Massilia 578 species were cultured out of precipitation by us previously (Failor et al., 2017) and were recently 579 found in rain and rain that had fallen through a forest canopy (Ladin et al., 2021). Finally, OTUs 580 and named species belonging to the genus Massilia have been found in plants, soil, and even 581 extreme environments (Ofek et al., 2012, Rastogi et al., 2012, Bodenhausen et al., 2013, Purahong et al., 2018, Singh et al., 2019, Holochová et al., 2020). Therefore, members of the genus Massilia 
583 may cycle through multiple environments and some of them may be transported by rain to leaf 584 surfaces where they colonize the phyllosphere.

Other rain-borne genera likely to colonize the tomato phyllosphere based on our data include Janthinobacterium, which, as the genus Massilia, is a member of the Burkholderiaceae plant but not in the tomato plant protected from rain in the ONT MinION experiment. However,

Janthinobacterium was not found at significantly higher relative abundance in tomato plants grown outside compared to greenhouse-grown tomatoes. Its inconsistent presence in rain may explain this result.

Finally, we found evidence for OTUs and named species of the genus Pseudomonas to 593 colonize tomato plants. Unexpectedly though, we did not find a single member of the 594 Pseudomonas syringae species complex ( $P$. syringae sensu lato), which includes many plant 595 pathogenic and commensal lineages (Vinatzer et al., 2016, Monteil et al., 2016). We do not have any good explanation why we neither found $P$. syringae at relatively high abundance in the analyzed rain samples nor in our phyllosphere samples treated with 100X-rain although we had 598 previously cultured $P$. syringae from rain (Failor et al., 2017) and from plants in our geographic 599 area (Clarke et al., 2010). We conclude that while $P$. syringae pathogens are present in rain and 600 disseminated by precipitation, they may not be a major component of precipitation microbiota, at 601 least not in the geographic area where the experiments here described were performed. Importantly though, while our data and the literature suggest that members of some common phyllosphere genera are rain-borne, the fact that we found different enrichment of OTUs

604 from these genera across experimental conditions, lab versus greenhouse, precluded the 605 identification of likely rain-borne tomato phyllosphere colonizers at the species level. One possible 
explanation for this is that each rain event harbored such different taxa and this increased variation coupled with low replication meant that OTUs with higher relative abundance on tomatoes grown

608 outside were simply missed in the lab experiments. Conversely, the small number of analyzed tomato plants grown outside exposed to rain made it difficult to find significant differences

610 compared to the tomatoes grown inside not exposed to rain. Finally, laboratory conditions may

611 not have allowed some of the OTUs found outside to effectively grow on tomato plants inside the

612 laboratory. On the other hand, artificial light and almost constant temperature and humidity may

613 have favored tomato colonization of related, but different, species compared to the most effective 614 colonizers of tomato plants grown outside, where plants are exposed to natural sunlight, including

615 UV radiation and dramatic temperature and humidity changes. These differences in environmental 616 conditions may explain why members of the genera Sphingomonas and Methylobacterium were

617 consistently found in rain and in significantly higher abundance on tomato plants grown outside

618 than in greenhouse-grown tomatoes but OTUs of these genera did not significantly increase in our

619 controlled laboratory experiments after 100X-rain treatments. Finally, the use of concentrated rain

620 microbiota instead of rain may have led to increased competition between rain-borne bacteria and

621 suppressed the colonization efficiency and growth of some while favoring the growth of others.

To follow up on the results reported here and to gain further insight into the relative

623 importance of seeds, soil, the atmosphere, and precipitation as reservoirs of phyllosphere

624 microbiota, we propose to expand the kind of controlled experiments we performed here but

625 growing tomatoes outdoors from either sterilized seeds or non-sterilized seeds, either not limiting

626 exposure to precipitation or limiting exposure to precipitation (for example, through the use of

627 mobile rain-out shelters), and growing plants in native versus sterile soil. Moreover, strain-level

628 metagenomics (Olm et al., 2021) of all reservoir microbiota and phyllosphere microbiota could 
629 provide the necessary strain-level resolution to identify which strains from which reservoirs are

630 the most important colonizers of the phyllosphere.

\section{DATA DEPOSITION}

634 Sequences and metadata are being deposited at NCBI under BioProject DPRJNA719680. All

635 read processing steps, bioinformatic workflows, and scripts used in this research are available on

636 GitHub (https://github.com/marcoeml/VinatzerLab-Mechan-rain-phyllosphere-microbiota-2020).

\section{FUNDING}

640 This research was supported by the National Science Foundation (DEB-1241068 and IOS-

641 1754721). Funding to BAV was also provided in part by the Virginia Agricultural Experiment

642 Station and the Hatch Program of the National Institute of Food and Agriculture, United States

643 Department of Agriculture.

\section{LITERATURE CITED}

646

647 Aho K, Weber CF, Christner BC, Vinatzer BA, Morris CE, Joyce R, Failor K, Werth JT, Bayless-

648 Edwards ALH, Schmale III DG, 2020. Spatiotemporal patterns of microbial composition and diversity in precipitation. Ecological Monographs 90 (1), e01394.

650 Allard SM, Ottesen AR, Micallef SA, 2020. Rain induces temporary shifts in epiphytic bacterial communities of cucumber and tomato fruit. Scientific Reports 10, 1765. 
652 Amato P, Joly M, Besaury L, Oudart A, Taib N, Moné AI, Deguillaume L, Delort A-M, Debroas D, 2017. Active microorganisms thrive among extremely diverse communities in cloud water. PLOS ONE 12, e0182869-e.

655 Amato P, Joly M, Schaupp C, Attard E, Möhler O, Morris CE, Brunet Y, Delort AM, 2015. 656 Survival and ice nucleation activity of bacteria as aerosols in a cloud simulation chamber. Atmos. Chem. Phys. 15, 6455-65.

Badri DV, Zolla G, Bakker MG, Manter DK, Vivanco JM, 2013. Potential impact of soil microbiomes on the leaf metabolome and on herbivore feeding behavior. New Phytologist

Berg M, Koskella B, 2018. Nutrient- and dose-dependent microbiome-mediated protection against a plant pathogen. Current Biology 28, 2487-92.e3. Leaves and the Roots of Arabidopsis thaliana. PLOS ONE 8, e56329.

Bolger AM, Lohse M, Usadel B, 2014. Trimmomatic: a flexible trimmer for Illumina sequence and Environmental Microbiology 35, 1231-2.

669 Bowers RM, Mcletchie S, Knight R, Fierer N, 2011. Spatial variability in airborne bacterial communities across land-use types and their relationship to the bacterial communities of

672 Brown CT, Irber L., 2016. sourmash: a library for MinHash sketching of DNA. Journal of Open $673 \quad$ Source Software (5), 1. 
674 Brown JKM, Hovmøller MS, 2002. Aerial dispersal of pathogens on the global and continental scales and Its impact on plant disease. Science 297, 537.

Bulgarelli D, Schlaeppi K, Spaepen S, Van Themaat EVL, Schulze-Lefert P, 2013. Structure and functions of the bacterial microbiota of plants. Annual Review of Plant Biology 64, 807-38. seasonal patterns in the airborne microbiome coupled to general and regional atmospheric

681 Caporaso JG, Kuczynski J, Stombaugh J, Bittinger K, Bushman FD, Costello EK, Fierer N, Peña

Christner BC, Morris CE, Foreman CM, Cai R, Sands DC, 2008. Ubiquity of biological ice nucleators in snowfall. Science 319, 1214. Naturally Lacking the Classical P. syringae hrp/hrc Locus Are Common Leaf Colonizers nucleation-active bacteria: The role of rain. Phytopathology 80, 934-7.

694 Copeland JK, Yuan L, Layeghifard M, Wang PW, Guttman DS, 2015. Seasonal community succession of the phyllosphere microbiome. Mol Plant Microbe Interact 28, 274-85. 
696 Coutinho TA, Venter SN, 2009. Pantoea ananatis: an unconventional plant pathogen. Molecular Plant Pathology 10, 325-35.

698 Durán P, Thiergart T, Garrido-Oter R, Agler M, Kemen E, Schulze-Lefert P, Hacquard S, 2018. Microbial interkingdom interactions in roots promote Arabidopsis survival. Cell 175, 973-

701 Failor KC, Schmale DG, 3rd, Vinatzer BA, Monteil CL, 2017. Ice nucleation active bacteria in precipitation are genetically diverse and nucleate ice by employing different mechanisms. The ISME Journal 11, 2740-53.

704 Fitzpatrick CR, Copeland J, Wang PW, Guttman DS, Kotanen PM, Johnson MTJ, 2018. Assembly and ecological function of the root microbiome across angiosperm plant species. Proceedings of the National Academy of Sciences 115, E1157.

Goh C-H, Veliz Vallejos DF, Nicotra AB, Mathesius U, 2013. The impact of beneficial plantassociated microbes on plant phenotypic plasticity. Journal of Chemical Ecology 39, 826-39.

709 Grady KL, Sorensen JW, Stopnisek N, Guittar J, Shade A, 2019. Assembly and seasonality of core phyllosphere microbiota on perennial biofuel crops. Nature Communications 10, 4135.

711 Graham BE, Stegen CJ, 2017. Dispersal-based microbial community assembly decreases biogeochemical function. Processes 5.

713 Hacquard S, Garrido-Oter R, González A, Spaepen S, Ackermann G, Lebeis S, Mchardy Alice c, 714 Dangl Jeffrey 1, Knight R, Ley R, Schulze-Lefert P, 2015. Microbiota and host nutrition across 715 plant and animal kingdoms. Cell Host \& Microbe 17, 603-16.

716 Hadley W, 2009. Ggplot2: Elegant graphics for data analysis. 
717 Hirano SS, Upper CD, 2000. Bacteria in the leaf ecosystem with emphasis on Pseudomonas syringae - a pathogen, ice nucleus, and epiphyte. Microbiology and molecular biology reviews 64, 624-53.

Hiraoka S, Miyahara M, Fujii K, Machiyama A, Iwasaki W, 2017. Seasonal analysis of microbial communities in precipitation in the greater Tokyo area, Japan. Frontiers in microbiology 8 ,

Holochová P, Mašlaňová I, Sedláček I, Švec P, Králová S, Kovařovic V, Busse H-J, Staňková E, Barták M, Pantůček R, 2020. Description of Massilia rubra sp. nov., Massilia aquatica sp. nov., Massilia mucilaginosa sp. nov., Massilia frigida sp. nov., and one Massilia

Jacobs JL, Sundin GW, 2001. Effect of solar UV-B radiation on a phyllosphere bacterial community. Applied and Environmental Microbiology 67, 5488.

Juul S, Izquierdo F, Hurst A, Dai X, Wright A, Kulesha E, Pettett R, Turner DJ, 2015. What's in

Kembel SW, O'connor TK, Arnold HK, Hubbell SP, Wright SJ, Green JL, 2014. Relationships between phyllosphere bacterial communities and plant functional traits in a neotropical forest. of metagenomic sequences. Genome research 26, 1721-9.

737 Knief C, Delmotte N, Chaffron S, Stark M, Innerebner G, Wassmann R, Von Mering C, Vorholt JA, 2012. Metaproteogenomic analysis of microbial communities in the phyllosphere and rhizosphere of rice. The ISME Journal 6, 1378-90. 
Ladin ZS, Ferrell B, Dums JT, Moore RM, Levia DF, Shriver WG, D’amico V, Trammell TLE, measuring microbial biodiversity within forest ecosystems. Scientific Reports 11, 1629.

Lahti L. SS, Et Al, 2020. Microbiome.

744 Lighthart B, Shaffer BT, 1995. Airborne bacteria in the atmospheric surface layer: Temporal distribution above a grass seed field. Applied and Environmental Microbiology 61, 1492.

Lindemann J, Constantinidou HA, Barchet WR, Upper CD, 1982. Plants as sources of airborne bacteria, including ice nucleation-active bacteria. Applied and Environmental Microbiology

Lindemann J, Upper CD, 1985. Aerial dispersal of epiphytic bacteria over bean plants. Applied and Environmental Microbiology 50, 1229-32.

751 Lindow SE, Brandl MT, 2003. Microbiology of the phyllosphere. Applied and Environmental Microbiology 69, 1875.

Love MI, Huber W, Anders S, 2014. Moderated estimation of fold change and dispersion for RNA-

Lu T, Ke M, Lavoie M, Jin Y, Fan X, Zhang Z, Fu Z, Sun L, Gillings M, Peñuelas J, Qian H, Zhu Y-G, 2018. Rhizosphere microorganisms can influence the timing of plant flowering.

Maignien L, Deforce EA, Chafee ME, Eren AM, Simmons SL, 2014. Ecological succession and stochastic variation in the assembly of Arabidopsis thaliana phyllosphere communities. mBio

Mcmurdie PJ, Holmes S, 2013. phyloseq: An R Package for reproducible interactive analysis and graphics of microbiome census data. PLOS ONE 8, e61217. 
763 Mechan Llontop ME, Hurley K, Tian L, Bernal Galeano VA, Wildschutte HK, Marine SC, Yoder

764 KS, Vinatzer BA, 2020. Exploring Rain as Source of Biological Control Agents for Fire $765 \quad$ Blight on Apple. Frontiers in microbiology 11.

766 Monteil CL, Bardin M, Morris CE, 2014. Features of air masses associated with the deposition of 767 Pseudomonas syringae and Botrytis cinerea by rain and snowfall. The ISME Journal 8, 2290$768 \quad 304$.

769 Monteil CL, Yahara K, Studholme DJ, Mageiros L, Méric G, Swingle B, Morris CE, Vinatzer BA, 770 Sheppard SK, 2016. Population-genomic insights into emergence, crop adaptation and 771 dissemination of Pseudomonas syringae pathogens. Microbial genomics 2, e000089-e.

772 Morris CE, 2002. Phyllosphere. In. eLS.

773 Morris CE, Conen F, Alex Huffman J, Phillips V, Pöschl U, Sands DC, 2014. Bioprecipitation: a 774 feedback cycle linking Earth history, ecosystem dynamics and land use through biological ice 775 nucleators in the atmosphere. Global Change Biology 20, 341-51.

776 Morris CE, Sands DC, Vinatzer BA, Glaux C, Guilbaud C, Buffiere A, Yan S, Dominguez H, 777 Thompson BM, 2008. The life history of the plant pathogen Pseudomonas syringae is linked 778 to the water cycle. Isme $j$ 2, 321-34.

779 Ofek M, Hadar Y, Minz D, 2012. Ecology of Root Colonizing Massilia (Oxalobacteraceae). PLOS $780 \quad O N E$ 7, e40117.

781 Oksanen J. BFG, Friendly M., Kindt R., Legendre P., Mcglinn D., Minchin P. R., O'hara R. B., 782 Simpson G. L., Solymos P., Stevens M. H., Szoecs E., Wagner H., 2020. vegan: Community 783 ecology package. 
Olm MR, Crits-Christoph A, Bouma-Gregson K, Firek BA, Morowitz MJ, Banfield JF, 2021. inStrain profiles population microdiversity from metagenomic data and sensitively detects shared microbial strains. Nature Biotechnology.

Ottesen AR, González Peña A, White JR, Pettengill JB, Li C, Allard S, Rideout S, Allard M, Hill Microbiology 13, 114

Ottesen AR, Gorham S, Reed E, Newell MJ, Ramachandran P, Canida T, Allard M, Evans P, Brown E, White JR, 2016. Using a control to better understand phyllosphere microbiota. PLOS ONE 11, e0163482.

Pérez-Jaramillo JE, De Hollander M, Ramírez CA, Mendes R, Raaijmakers JM, Carrión VJ, 2019. vulgaris) in native and agricultural soils from Colombia. Microbiome 7, 114.

Peter H, Hörtnagl P, Reche I, Sommaruga R, 2014. Bacterial diversity and composition during rain events with and without Saharan dust influence reaching a high mountain lake in the Alps. Environmental microbiology reports 6, 618-24.

802 Powell JR, Karunaratne S, Campbell CD, Yao H, Robinson L, Singh BK, 2015. Deterministic processes vary during community assembly for ecologically dissimilar taxa. Nature

Purahong W, Orrù L, Donati I, Perpetuini G, Cellini A, Lamontanara A, Michelotti V, Tacconi G, 
Pseudomonas syringae pv. actinidiae Infection Shaping Bacterial Phyllosphere Communities of Kiwifruit Plants. Frontiers in Plant Science 9.

809 Quast C, Pruesse E, Yilmaz P, Gerken J, Schweer T, Yarza P, Peplies J, Glöckner FO, 2013. The SILVA ribosomal RNA gene database project: improved data processing and web-based tools. Nucleic acids research 41, D590-D6.

812 Rastogi G, Sbodio A, Tech JJ, Suslow TV, Coaker GL, Leveau JHJ, 2012. Leaf microbiota in an agroecosystem: spatiotemporal variation in bacterial community composition on field-grown lettuce. The ISME Journal 6, 1812-22.

815 Ritpitakphong U, Falquet L, Vimoltust A, Berger A, Métraux J-P, L'haridon F, 2016. The Phytologist 210, 1033-43.

818 Šantl-Temkiv T, Finster K, Dittmar T, Hansen BM, Thyrhaug R, Nielsen NW, Karlson UG, 2013.

819 Hailstones: A window into the microbial and chemical inventory of a storm cloud. PLOS ONE $820 \quad 8$, e53550.

821 Schmale DG, Ross SD, 2015. Highways in the sky: Scales of atmospheric transport of plant pathogens. Annual Review of Phytopathology 53, 591-611.

823 Singh P, Santoni S, Weber A, This P, Péros J-P, 2019. Understanding the phyllosphere microbiome assemblage in grape species (Vitaceae) with amplicon sequence data structures. Scientific Reports 9, 14294.

826 Toju H, Okayasu K, Notaguchi M, 2019. Leaf-associated microbiomes of grafted tomato plants. 
828 Torres-Cortés G, Bonneau S, Bouchez O, Genthon C, Briand M, Jacques M-A, Barret M, 2018. Functional microbial features driving community assembly during seed germination and emergence. Frontiers in Plant Science 9.

831 Vacher C, Hampe A, Porté AJ, Sauer U, Compant S, Morris CE, 2016. The phyllosphere: 832 Microbial jungle at the plant-climate interface. Annual Review of Ecology, Evolution, and $833 \quad$ Systematics 47, 1-24.

834 Vaïtilingom M, Attard E, Gaiani N, Sancelme M, Deguillaume L, Flossmann AI, Amato P, Delort A-M, 2012. Long-term features of cloud microbiology at the puy de Dôme (France).

Van Bruggen AHC, Goss EM, Havelaar A, Van Diepeningen AD, Finckh MR, Morris JG, 2019. One Health - Cycling of diverse microbial communities as a connecting force for soil, plant, animal, human and ecosystem health. Science of The Total Environment 664, 927-37.

840 Vinatzer BA, Weisberg AJ, Monteil CL, Elmarakeby HA, Sheppard SK, Heath LS, 2016. A Proposal for a Genome Similarity-Based Taxonomy for Plant-Pathogenic Bacteria that Is

844 Vorholt JA, 2012. Microbial life in the phyllosphere. Nature Reviews Microbiology 10, 828-40.

845 Wagner MR, Lundberg DS, Del Rio TG, Tringe SG, Dangl JL, Mitchell-Olds T, 2016. Host genotype and age shape the leaf and root microbiomes of a wild perennial plant. Nature Communications 7, 12151 -.

Walterson AM, Stavrinides J, 2015. Pantoea: insights into a highly versatile and diverse genus within the Enterobacteriaceae. FEMS Microbiology Reviews 39, 968-84. 
850 Weiss S, Xu ZZ, Peddada S, Amir A, Bittinger K, Gonzalez A, Lozupone C, Zaneveld JR,

851 Vázquez-Baeza Y, Birmingham A, Hyde ER, Knight R, 2017. Normalization and microbial

852 differential abundance strategies depend upon data characteristics. Microbiome 5, 27.

853 Williams TR, Moyne A-L, Harris LJ, Marco ML, 2013. Season, irrigation, leaf age, and

854 Escherichia coli inoculation influence the bacterial diversity in the lettuce phyllosphere.

$855 \quad$ PLOS ONE 8, e68642.

856 Woo C, An C, Xu S, Yi S-M, Yamamoto N, 2018. Taxonomic diversity of fungi deposited from

857 the atmosphere. The ISME Journal 12, 2051-60.

858 Woo C, Yamamoto N, 2020. Falling bacterial communities from the atmosphere. Environmental Microbiome 15, 22.

860 Zarraonaindia I, Owens SM, Weisenhorn P, West K, Hampton-Marcell J, Lax S, Bokulich NA,

861 Mills DA, Martin G, Taghavi S, Van Der Lelie D, Gilbert JA, 2015. The soil microbiome 862 influences grapevine-associated microbiota. mBio 6, e02527-14.

863 Zhou J, Ning D, 2017. Stochastic community assembly: Does it matter in microbial ecology? Microbiology and Molecular Biology Reviews 81, e00002-17. 


\section{TABLES}

867 Table 1. Metadata for analyzed rain samples.

868

\begin{tabular}{ccccc}
\hline $\begin{array}{c}\text { Rain } \\
\text { collection ID }\end{array}$ & Month & Year & $\begin{array}{c}\text { \# raw } \\
\text { reads }\end{array}$ & $\begin{array}{c}\text { used for plant } \\
\text { inoculations }\end{array}$ \\
\hline VTP049 & April & 2015 & 7,133 & Yes \\
VTP050-1 & August & 2015 & 75,555 & Yes \\
VTP050-2 & August & 2015 & 68,612 & Yes \\
VTP061 & March & 2016 & 75,166 & Yes \\
VTP062 & April & 2016 & 7,533 & Yes \\
VTP063 & May & 2016 & 27,729 & Yes \\
VTP064 & July & 2016 & 142,736 & Yes \\
VTP065 & October & 2016 & 35,216 & No \\
VTP066 & December & 2016 & 32,866 & No \\
VTP2019 & June & 2019 & 419,596 & No \\
\hline
\end{tabular}

869

870 
871 Table 2. Species with highest relative abundance on tomato plants on day-7 after treatment with 872 100X-rain based on metagenomic sequencing.

873

\begin{tabular}{|c|c|c|c|c|c|}
\hline species & $\begin{array}{c}\# \\
\text { positive } \\
\text { day } 7 \\
\text { samples }^{1}\end{array}$ & $\begin{array}{c}\text { average } \\
\text { abundance } \\
\text { in rain }^{2}\end{array}$ & $\begin{array}{c}\text { average } \\
\text { abundance } \\
\text { on day } 0^{2}\end{array}$ & $\begin{array}{c}\text { average } \\
\text { abundance } \\
\text { on day } 7^{2}\end{array}$ & $\begin{array}{c}\text { highest } \\
\text { abundance } \\
\text { on day } 7\end{array}$ \\
\hline Pantoea vagans & 4 & 0.09 & 0.01 & 2.86 & 8.69 \\
\hline Pantoea agglomerans & 3 & 0.03 & 0.01 & 2.12 & 4.25 \\
\hline Novosphingobium resinovorum & 1 & 0.00 & 0.00 & 1.54 & 1.54 \\
\hline Pseudomonas citronellolis & 2 & 0.01 & 0.08 & 1.21 & 1.98 \\
\hline Buttiauxella sp. & 2 & 0.01 & 0.03 & 0.79 & 1.49 \\
\hline Janthinobacterium sp. & 1 & 0.01 & 0.01 & 0.77 & 0.77 \\
\hline Pseudomonas fluorescens & 4 & 0.03 & 0.02 & 0.75 & 1.16 \\
\hline Erwinia gerundensis & 2 & 0.01 & 0.01 & 0.75 & 1.31 \\
\hline $\begin{array}{l}\text { Massilia putida } \\
\text { Janthinobacterium }\end{array}$ & 3 & 0.01 & 0.01 & 0.63 & 0.76 \\
\hline agaricidamnosum & 1 & 0.01 & 0.00 & 0.62 & 0.62 \\
\hline Cedecea neteri & 2 & 0.01 & 0.02 & 0.53 & 1.00 \\
\hline Pseudomonas orientalis & 2 & 0.00 & 0.00 & 0.53 & 0.79 \\
\hline Massilia sp. & 4 & 0.01 & 0.02 & 0.45 & 0.90 \\
\hline Flavobacterium sp. HYN0086 & 1 & 0.06 & 0.02 & 0.41 & 0.41 \\
\hline Flavobacterium anhuiense & 1 & 0.08 & 0.04 & 0.40 & 0.40 \\
\hline Flavobacterium sp. & 1 & 0.04 & 0.02 & 0.33 & 0.33 \\
\hline Pseudomonas azotoformans & 4 & 0.01 & 0.00 & 0.31 & 0.48 \\
\hline Pseudomonas sp. & 2 & 0.00 & 0.00 & 0.27 & 0.33 \\
\hline Pseudomonas putida & 1 & 0.02 & 0.01 & 0.27 & 0.27 \\
\hline Enterobacter sp. & 1 & 0.00 & 0.01 & 0.26 & 0.26 \\
\hline Klebsiella michiganensis & 1 & 0.00 & 0.01 & 0.25 & 0.25 \\
\hline Pseudomonas protegens & 1 & 0.00 & 0.02 & 0.25 & 0.25 \\
\hline Pantoea ananatis & 1 & 0.00 & 0.00 & 0.24 & 0.24 \\
\hline Pseudomonas trivialis & 1 & 0.00 & 0.00 & 0.24 & 0.24 \\
\hline Pseudomonas rhizosphaerae & 2 & 0.01 & 0.01 & 0.23 & 0.27 \\
\hline Brevundimonas sp. & 1 & 0.01 & 0.00 & 0.23 & 0.23 \\
\hline Pseudomonas veronii & 1 & 0.00 & 0.00 & 0.23 & 0.23 \\
\hline Pseudomonas syringae & 1 & 0.01 & 0.02 & 0.22 & 0.22 \\
\hline Enterobacter cloacae & 2 & 0.02 & 0.01 & 0.15 & 0.24 \\
\hline Escherichia coli & 3 & 0.14 & 0.12 & 0.15 & 0.22 \\
\hline Sphingobium sp. & 1 & 0.00 & 0.00 & 0.15 & 0.15 \\
\hline Hyphomicrobium sp. & 1 & 0.01 & 0.00 & 0.13 & 0.13 \\
\hline Paraburkholderia fungorum & 1 & 0.00 & 0.00 & 0.11 & 0.11 \\
\hline
\end{tabular}


bioRxiv preprint doi: https://doi.org/10.1101/2021.04.08.438997; this version posted April 9, 2021. The copyright holder for this preprint (which was not certified by peer review) is the author/funder, who has granted bioRxiv a license to display the preprint in perpetuity. It is made available under ACC-BY-ND 4.0 International license.

\section{4}

\begin{tabular}{l} 
Staphylococcus aureus \\
Pseudomonas sp. \\
Pseudomonas sp. \\
\hline${ }^{1}$ out of 5 \\
2 only considering experiments \\
for which the species was \\
present on day 7
\end{tabular}

875 
876 Table 3. Taxonomic classification of ONT MinION reads using WIMP of a rain sample, a 877 tomato plant exposed to rain, and a tomato plant not exposed to rain.

\begin{tabular}{|c|c|c|c|}
\hline Taxon $^{1}$ & rain & $\begin{array}{c}\text { tomato exposed } \\
\text { to rain }\end{array}$ & $\begin{array}{c}\text { tomato not } \\
\text { exposed to rain }\end{array}$ \\
\hline Massilia putida & 2,461 & 165 & 0 \\
\hline Massilia sp. WG5 & 2,794 & 119 & 0 \\
\hline Deinococcus gobiensis & 0 & 67 & 1 \\
\hline Deinococcus actinosclerus & 0 & 56 & 2 \\
\hline Roseateles depolymerans & 33 & 55 & 0 \\
\hline Pantoea agglomerans & 659 & 45 & 1 \\
\hline Methylobacterium sp. PR1016A & 37 & 32 & 0 \\
\hline Acidovorax sp. KKS102 & 17 & 32 & 0 \\
\hline Pseudomonas oryzihabitans & 25 & 25 & 2 \\
\hline Variovorax paradoxus & 98 & 22 & 0 \\
\hline Phycomyces blakesleeanus & 15 & 22 & 29 \\
\hline Mitsuaria sp. 7 & 50 & 19 & 1 \\
\hline Pantoea vagans & 18 & 18 & 1 \\
\hline Methylobacterium phyllosphaerae & 75 & 18 & 1 \\
\hline Methylobacterium aquaticum & 42 & 17 & 0 \\
\hline Microbacterium testaceum & 0 & 16 & 0 \\
\hline Deinococcus soli Cha et al. 2016 & 0 & 15 & 0 \\
\hline Rubrivivax gelatinosus & 47 & 15 & 0 \\
\hline Massilia sp. B2 & 826 & 14 & 1 \\
\hline Rhizobacter gummiphilus & 53 & 13 & 0 \\
\hline Microbacterium sp. BH-3-3-3 & 12 & 12 & 1 \\
\hline Methylobacterium extorquens & 1,102 & 11 & 2 \\
\hline Pantoea sp. PSNIH1 & 0 & 10 & 1 \\
\hline Massilia sp. NR 4-1 & 550 & 9 & 1 \\
\hline Janthinobacterium sp. 1_2014MBL_MicDiv & 384 & 9 & 0 \\
\hline Janthinobacterium sp. LM6 & 350 & 9 & 1 \\
\hline Ralstonia solanacearum & 150 & 9 & 0 \\
\hline Janthinobacterium agaricidamnosum & 338 & 8 & 0 \\
\hline Sphingomonas taxi & 627 & 7 & 0 \\
\hline Janthinobacterium svalbardensis & 265 & 6 & 0 \\
\hline Modestobacter marinus & 162 & 5 & 3 \\
\hline Herbaspirillum frisingense & 199 & 4 & 0 \\
\hline Pseudomonas syringae & 165 & 4 & 0 \\
\hline Pseudomonas fluorescens & 10,173 & 3 & 0 \\
\hline Geodermatophilus obscurus & 163 & 3 & 0 \\
\hline
\end{tabular}


Pseudomonas protegens

Pseudomonas putida

906

289

${ }^{1}$ Only species with at least two reads in either the tomato plant exposed to rain or the tomato plant not exposed to rain are listed ranked from high to low by the number of reads in the tomato plant exposed to rain. Species with a higher number of reads in the tomato plant exposed to rain are in bold.

\section{FIGURE LEGENDS}

Figure 1. Bacterial population size in the phyllosphere of tomato plants A) grown exposed to rain in plastic pots at the Virginia Tech Kentland Farm compared to plants grown inside the laboratory B) grown on the roof of the Latham Hall research building exposed to rain compared to plants grown under laboratory conditions, and C) grown inside the laboratory seven days after being either treated with rain or autoclaved rain. T-test, $\mathrm{P}<0.001$.

Figure 2. Relative abundance (RA) of bacterial taxa in rainfall collected in Blacksburg, VA, on 9

894 different days in 2015, 2016, and 2019. Samples were rarefied to 6,419 sequences. A) RA at the

895 class level (only classes with a RA above 1\% are shown), B) RA at the genus level (only generated with RA above $2 \%$ are shown). For August 15, results are based on two technical replicates (see

897 also Table 1).

Figure 3. Alpha diversity measurements for rain compared to treated plants at day-0 vs day-7.

900 Three measures of alpha diversity (observed OTUs, Shannon diversity index, and Simpson

901 diversity index) were used. For rain, only samples used for plant inoculations were included. 
903 Figure 4. Principal coordinates analysis (PCoA) derived from weighted UniFrac distances (A),

904 unweighted UniFrac distances (B), and the dissimilarity matrix of Bray-Curtis (C). Only rain

905 samples used for plant inoculation were included. For experiments for which replicates were

906 available, the replicate samples are labeled as "monthyear.1" and "monthyear.2".

908 Figure 5. Relative abundance (RA) of bacterial taxa of plants treated with either 100X-rain,

909 filtered rain, or sterile water, at day-0 and day-7. Experiments are listed by dates from left to

910 right (April ‘15, August ‘15, March '16, April ‘16, May '16, and July '16). A) RA at the phylum

911 level (abundance $>1 \%$ ), B) . RA at the genus level (abundance $>3 \%$ ). For experiments for

912 which replicates were available relative abundance is based on all replicates.

913

914 Figure 6: Differential abundance analysis at the level of Operational Taxonomic Unit (OTU) using

915 DESeq2 (Love et al., 2014). The fold change is shown on the $\mathrm{X}$ axis and genera are listed on the

916 Y axis. Each colored dot represents a separate OTU. A) Comparison of phyllosphere microbiota

917 of plants treated with 100X-rain between day-0 and day-7, B) Comparison between rain microbiota

918 and phyllosphere microbiota 7 days after treatment with the respective 100X-rain sample.

920 Figure 7. Relative abundance of a representative selection of rain-borne genera that either failed 921 or succeeded in colonizing the tomato phyllosphere. Dates of experiments are listed on the $\mathrm{X}$ axis

922 of panels $\mathrm{G}$ and $\mathrm{H}$. Relative abundance is shown on the $\mathrm{Y}$ axis for rain, tomato plants on day-0 and 923 day-7 after being treated with 100X-rain (Rain), filtered rain, or sterile water (see panel B for 924 figure legend). A) Pantoea, B) Flavobacterium, C) Janthinobacterium, D) Pseudomonas, E) 925 Massilia, F) Methylobacterium, G) Acidiphilium, and H) Bacillus. 
927 Figure 8. Taxonomic composition and Alpha diversity of phyllosphere microbiota of tomato

928 plants grown hydroponically or in soil (both in a commercial greenhouse) and of tomato plants

929 grown outside (on the roof of the Latham Hall research building). A) Relative abundance at the

930 phylum level (only phyla with a RA above 1\% are shown), B) Alpha diversity (observed OTUs,

931 Shannon diversity index, and Simpson diversity index).

932

933 Figure 9. Differential abundance analysis at the level of Operational Taxonomic Unit (OTU)

934 using DESeq2 (Love et al., 2014). The fold change is shown on the $\mathrm{X}$ axis and genera are listed

935 on the Y axis. Each colored dot represents a separate OTU. A) Plants grown hydroponically in a

936 greenhouse compared with plants grown on the roof of the Latham Hall research building, B)

937 Plants grown in soil in a greenhouse compared with plants grown on the roof of the Latham Hall

938 research building.

\section{Supplementary Figures}

941

942 Supplementary Figure 1. Rarefaction curves of rain microbiota and tomato phyllosphere

943 microbiota treated with 100X-rain, filtered rain, or sterile water on day-0 and day-7.

945 Supplementary Figure 2. The core rain microbiome. Rain-associated OTUs at a detection 946 threshold of $0.1 \%$ and $100 \%$ as a prevalence threshold. 
948 Supplementary Figure 3. Differential abundance analysis at the OTU level usingDESeq2 (Love

949 et al., 2014). A) Comparison of rain microbiota with the tomato phyllosphere treated with sterile

950 water at day-0 during the same experiment, B) Comparison of rain microbiota with the tomato

951 phyllosphere microbiota on the day there were treated with 100X-rain (day-0).

952 
A

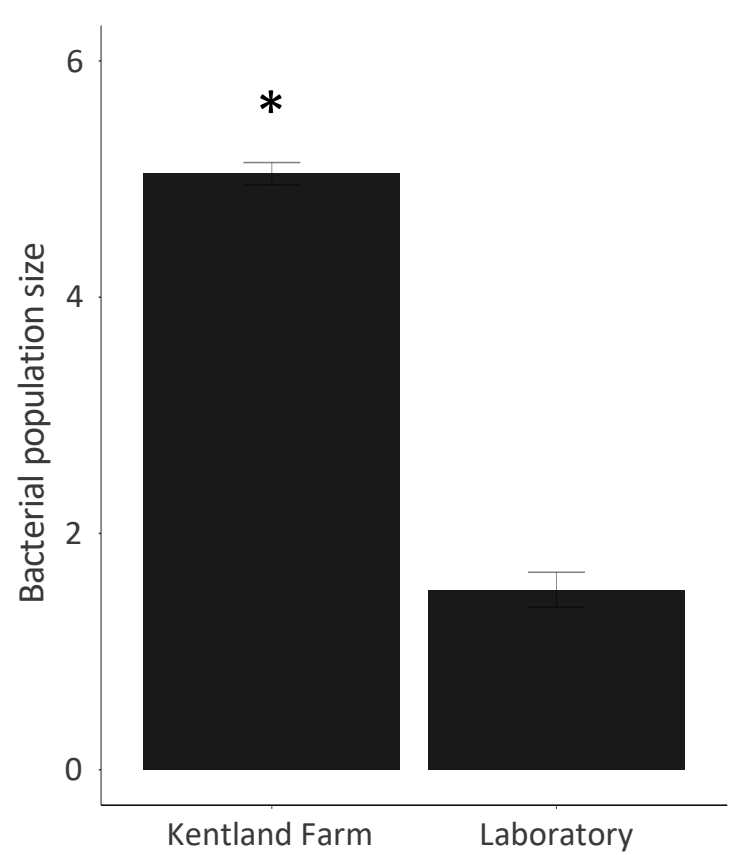

B

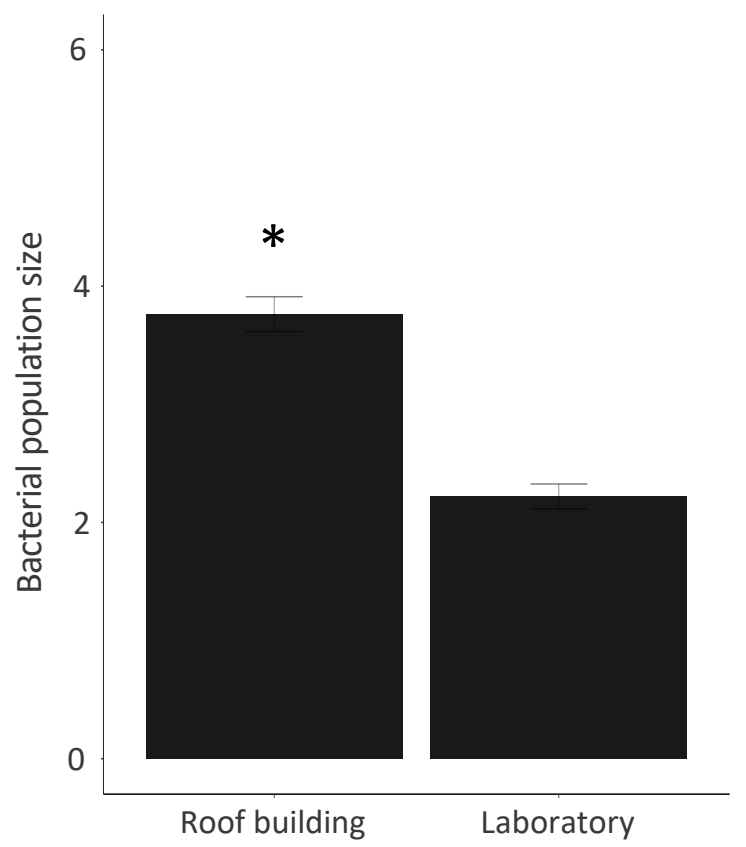

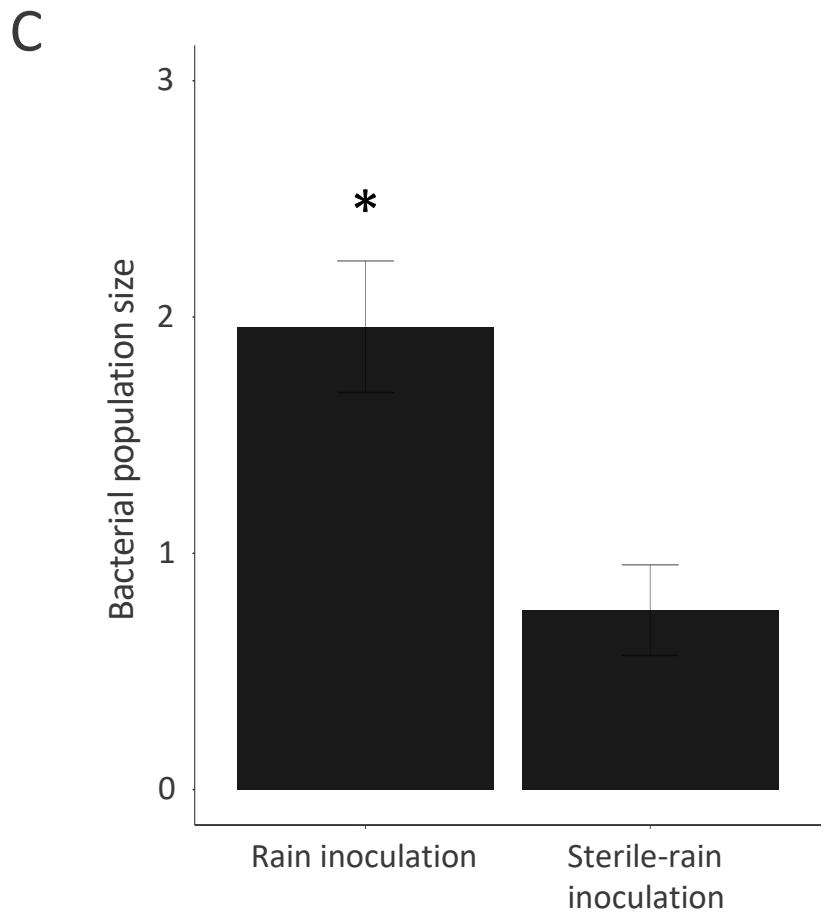

Figure 1. Bacterial population size in the phyllosphere of tomato plants $A$ ) grown exposed to rain in plastic pots at the Virginia Tech Kentland Farm compared to plants grown inside the laboratory B) grown on the roof of the Latham Hall research building exposed to rain compared to plants grown under laboratory conditions, and $\mathrm{C}$ ) grown inside the laboratory seven days after being either treated with rain or autoclaved rain. T-test, $\mathrm{P}<0.001$. 
A

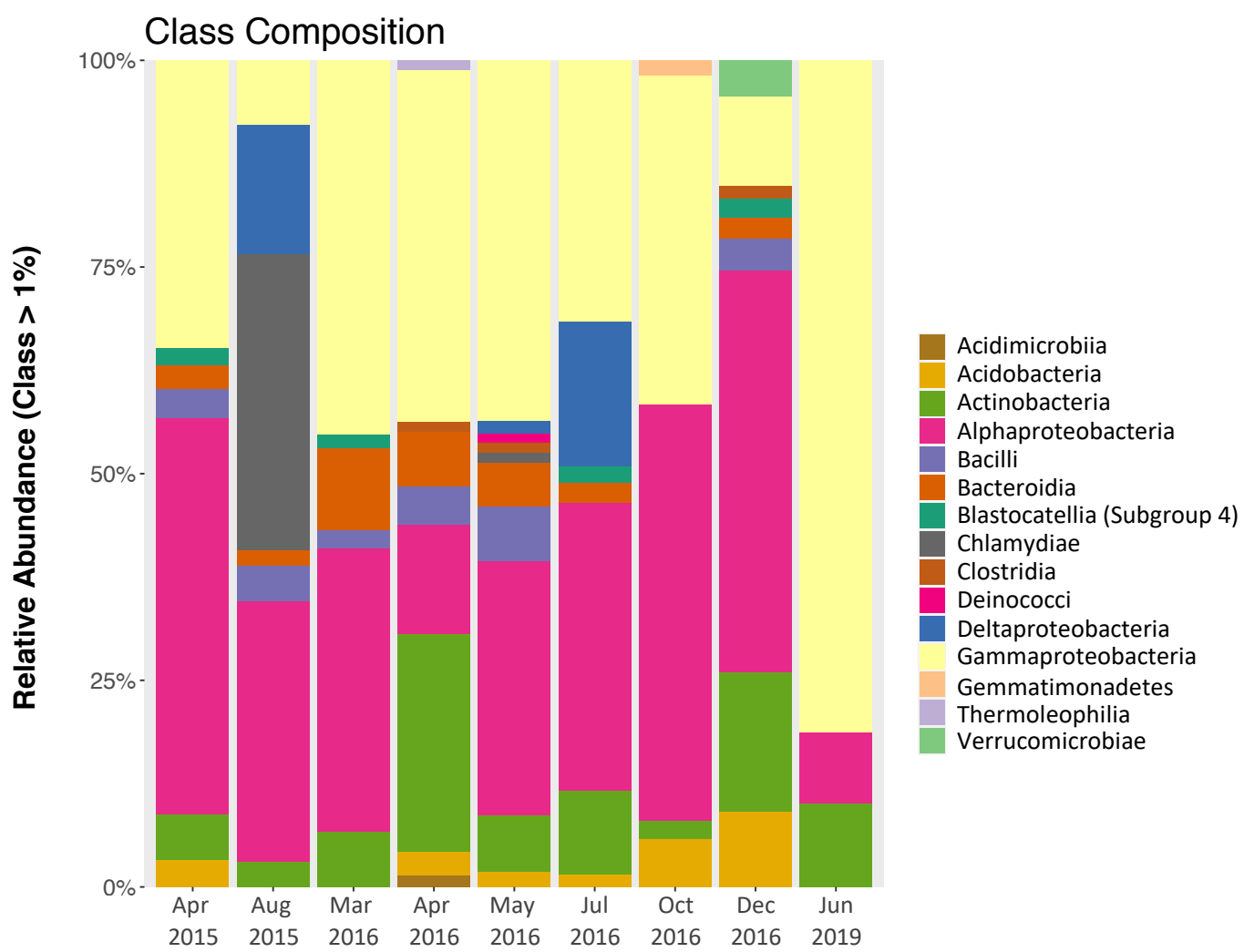

Rain collection event

B

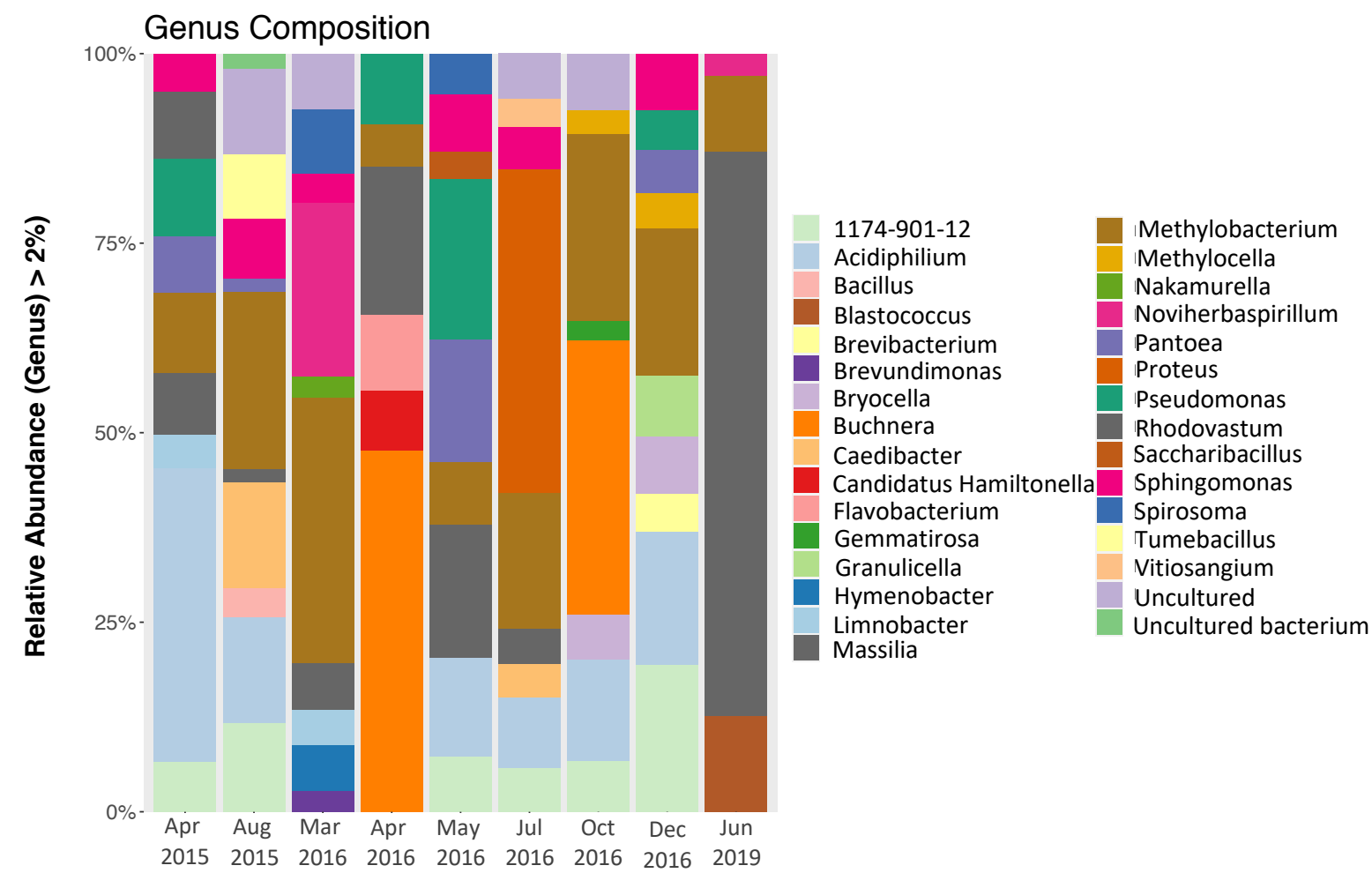

Rain collection event

Figure 2. Relative abundance (RA) of bacterial taxa in rainfall collected in Blacksburg, VA, on 9 different days in 2015, 2016, and 2019. Samples were rarefied to 6,419 sequences. A) RA at the class level (only classes with a RA above $1 \%$ are shown), B) RA at the genus level (only generated with RA above $2 \%$ are shown). For August 15, results are based on two technical replicates (see also Table 1). 


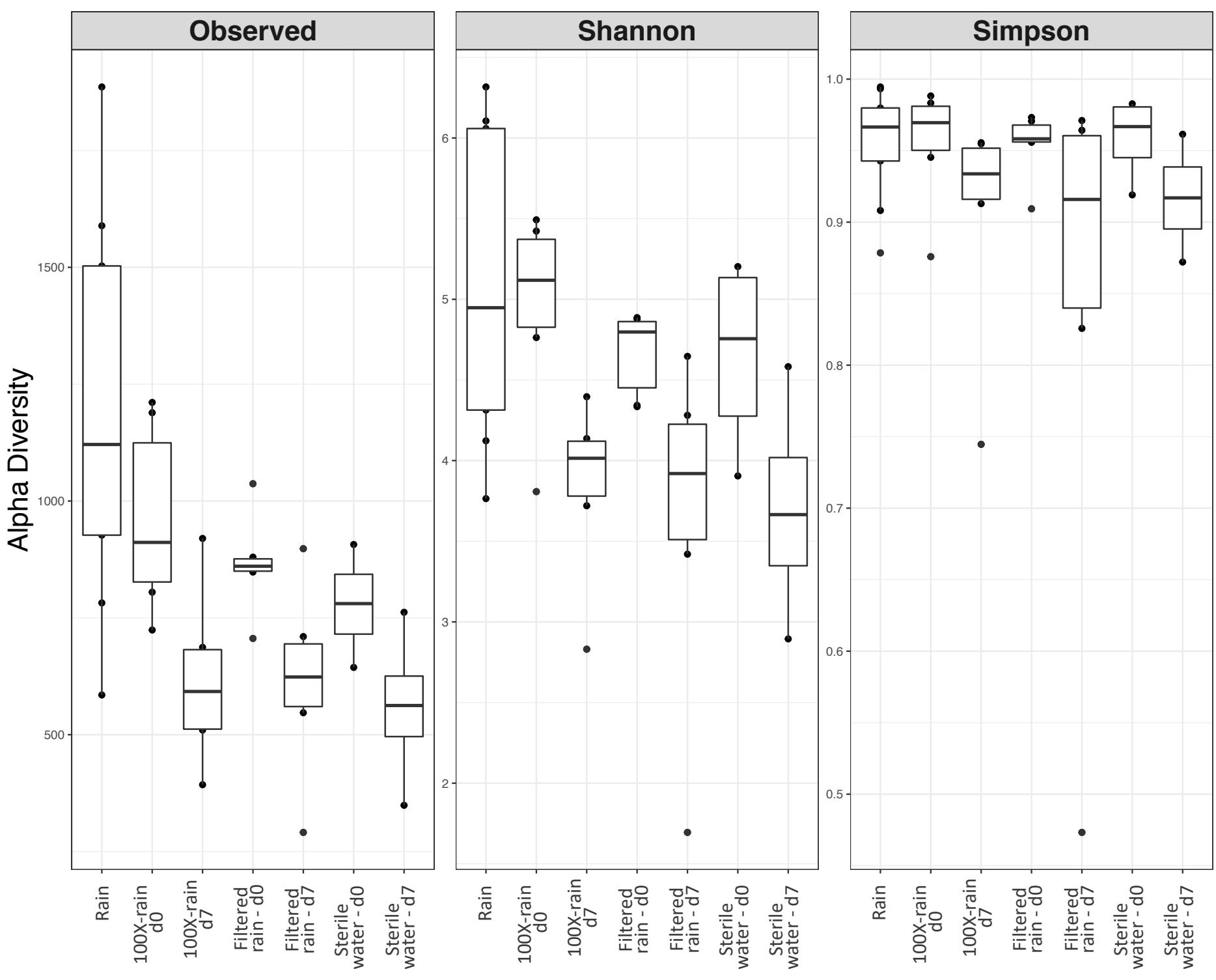

Figure 3. Alpha diversity measurements for rain compared to treated plants at day-0 vs day-7. Three measures of alpha diversity (observed OTUs, Shannon diversity index, and Simpson diversity index) were used. For rain, only samples used for plant inoculations were included. 

was not certified by peer review) is the author/funder, who has granted bioRxiv a license to display the preprint in perpetuity. It is made
available under aCC-BY-ND 4.0 International license.

A

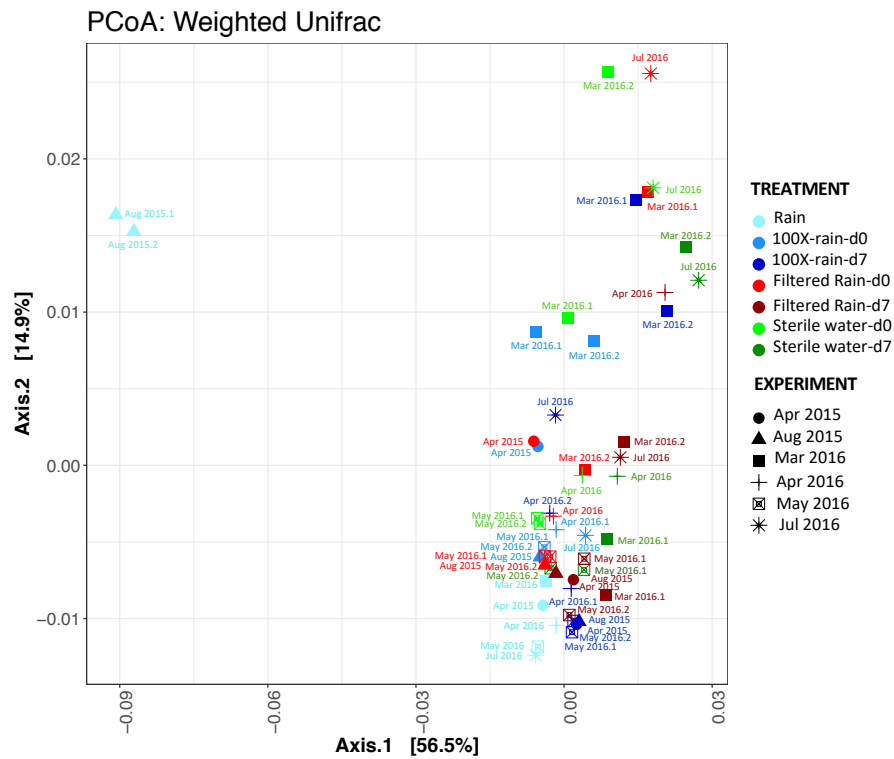

B

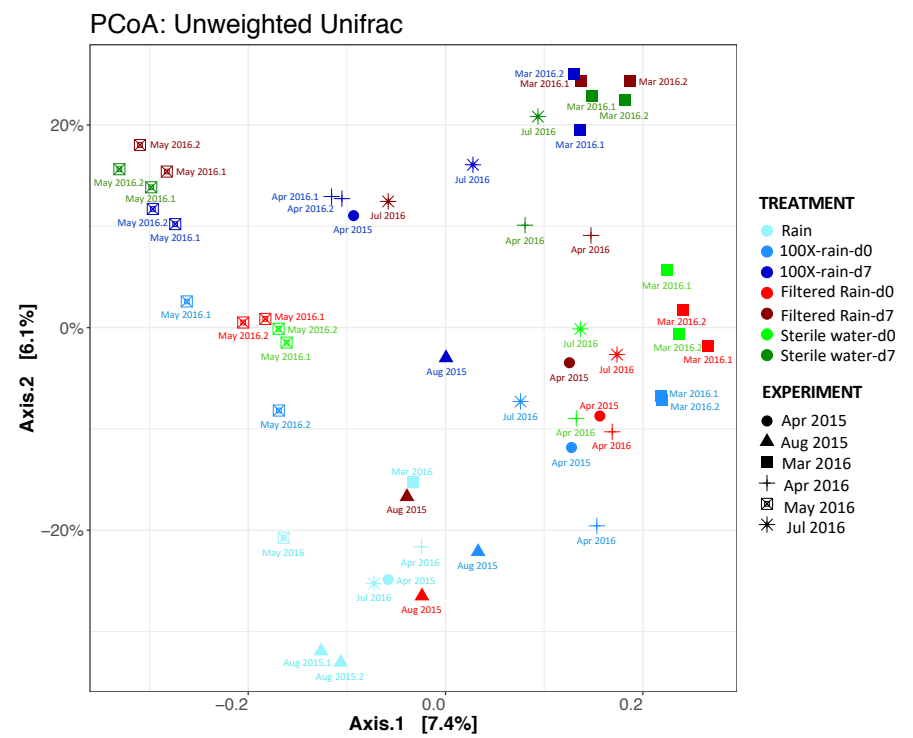

C

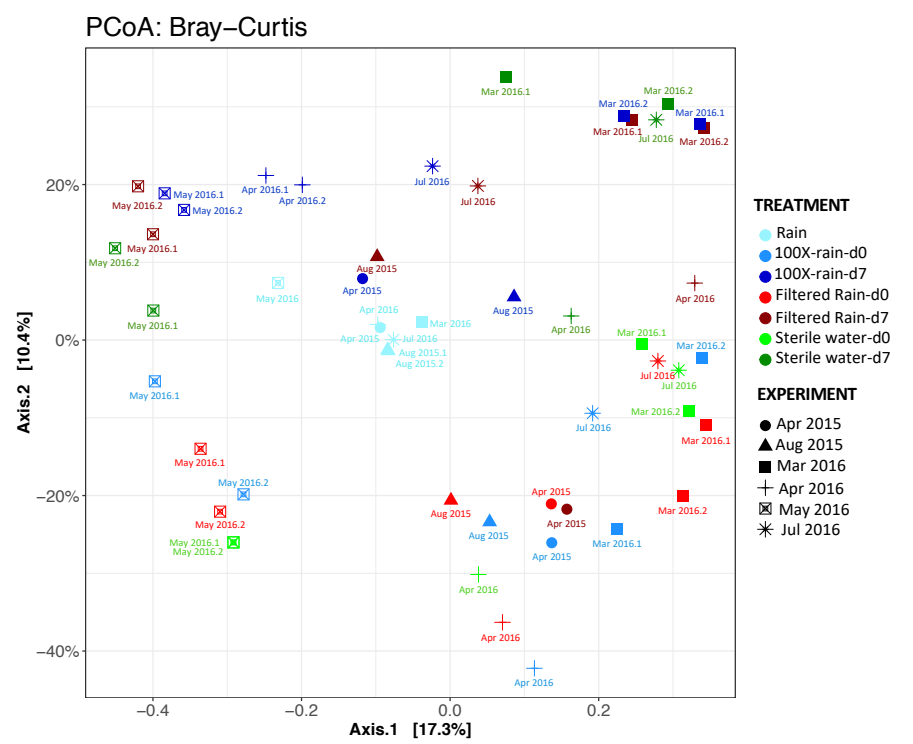

Figure 4. Principal coordinates analysis (PCOA) derived from weighted UniFrac distances (A), unweighted UniFrac distances (B), and the dissimilarity matrix of Bray-Curtis (C). Only rain samples used for plant inoculation were included. For experiments for which replicates were available, the replicate samples are labeled as "monthyear.1" and "monthyear.2". 


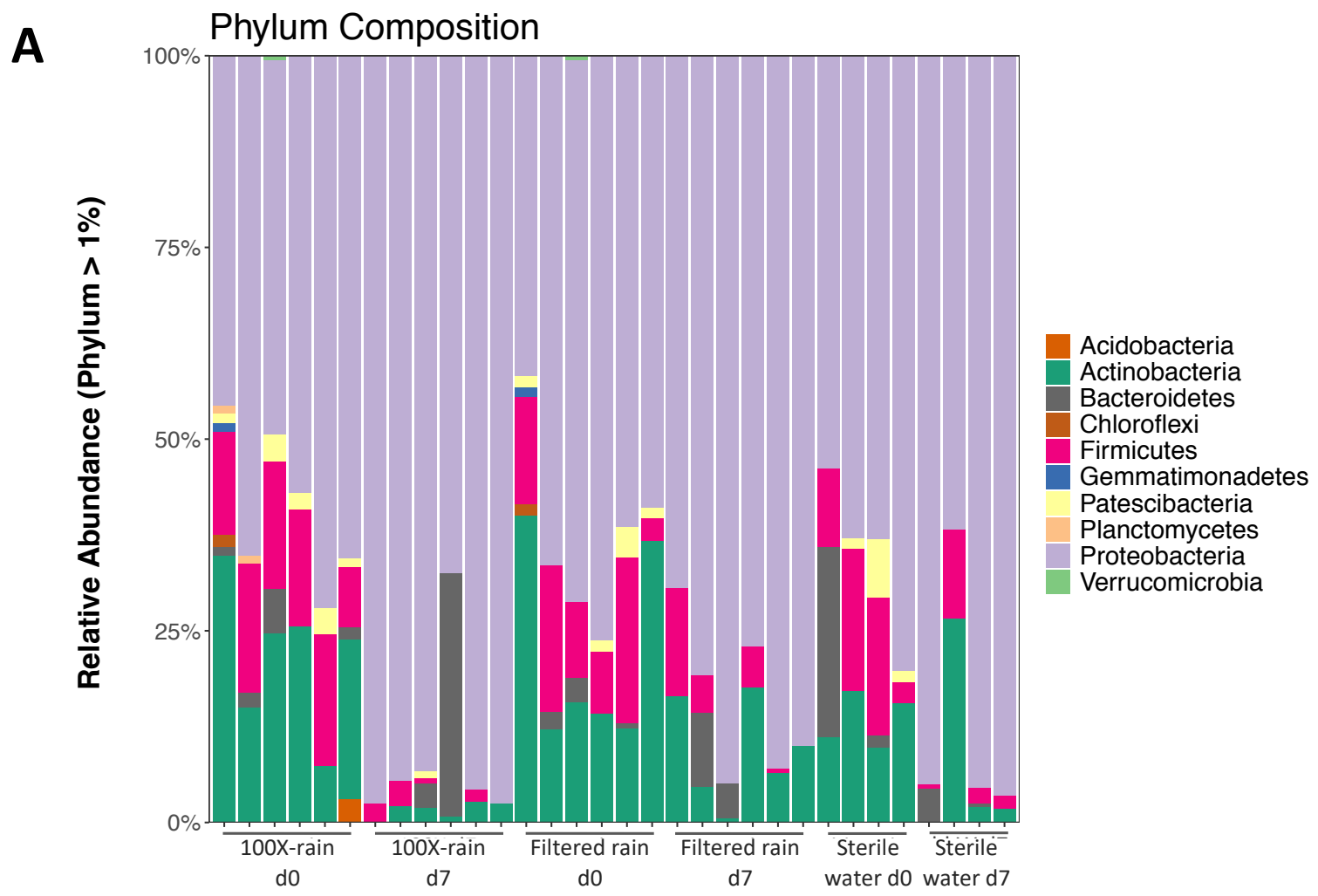

B

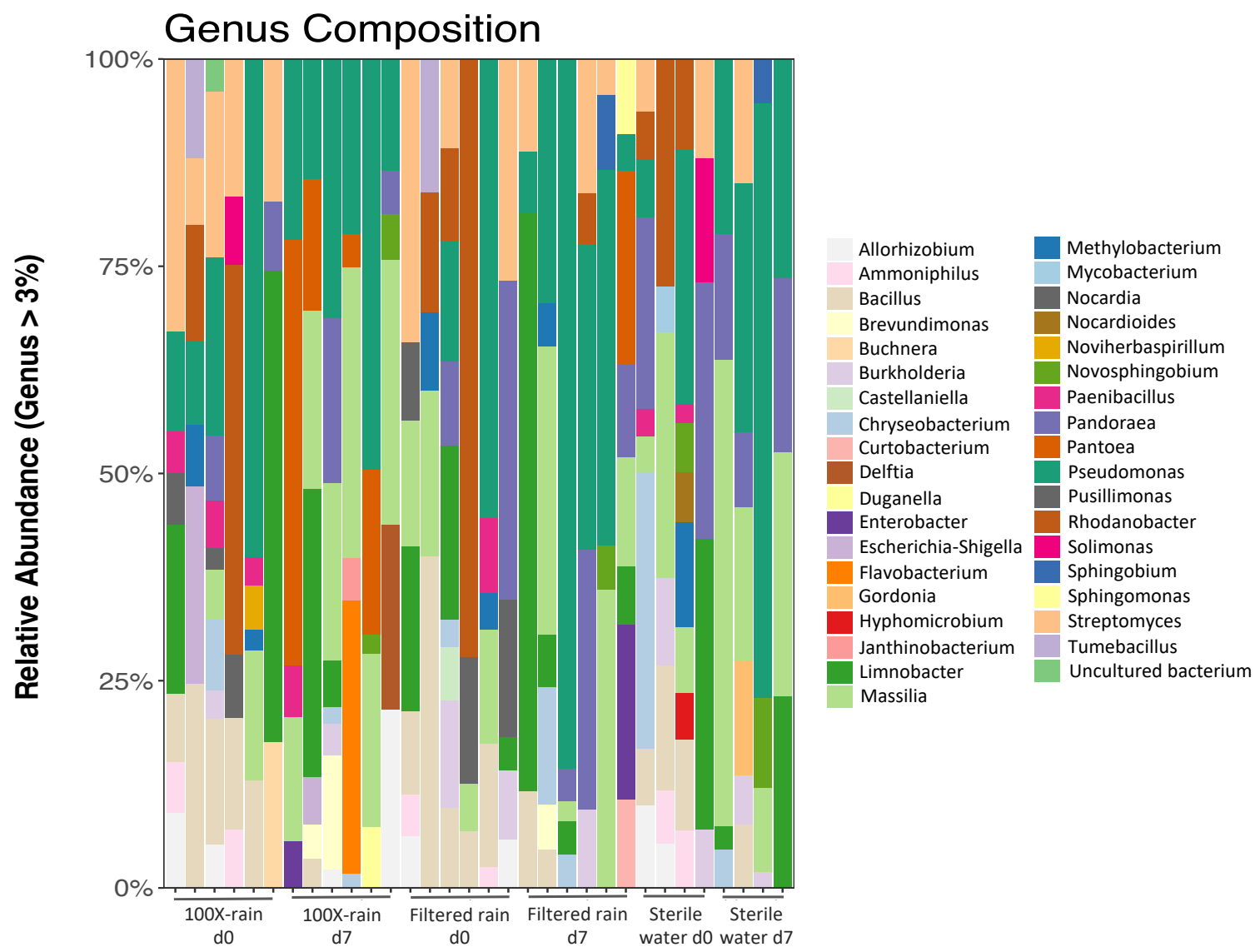

Figure 5. Relative abundance (RA) of bacterial taxa of plants treated with either 100X-rain, filtered rain, or sterile water, at day-0 and day-7. Experiments are listed by dates from left to right (April '15, August '15, March '16, April '16, May '16, and July '16). A) RA at the phylum level (abundance > 1\%), B). RA at the genus level (abundance $>3 \%$ ). For experiments for which replicates were available relative abundance is based on all replicates. 
A

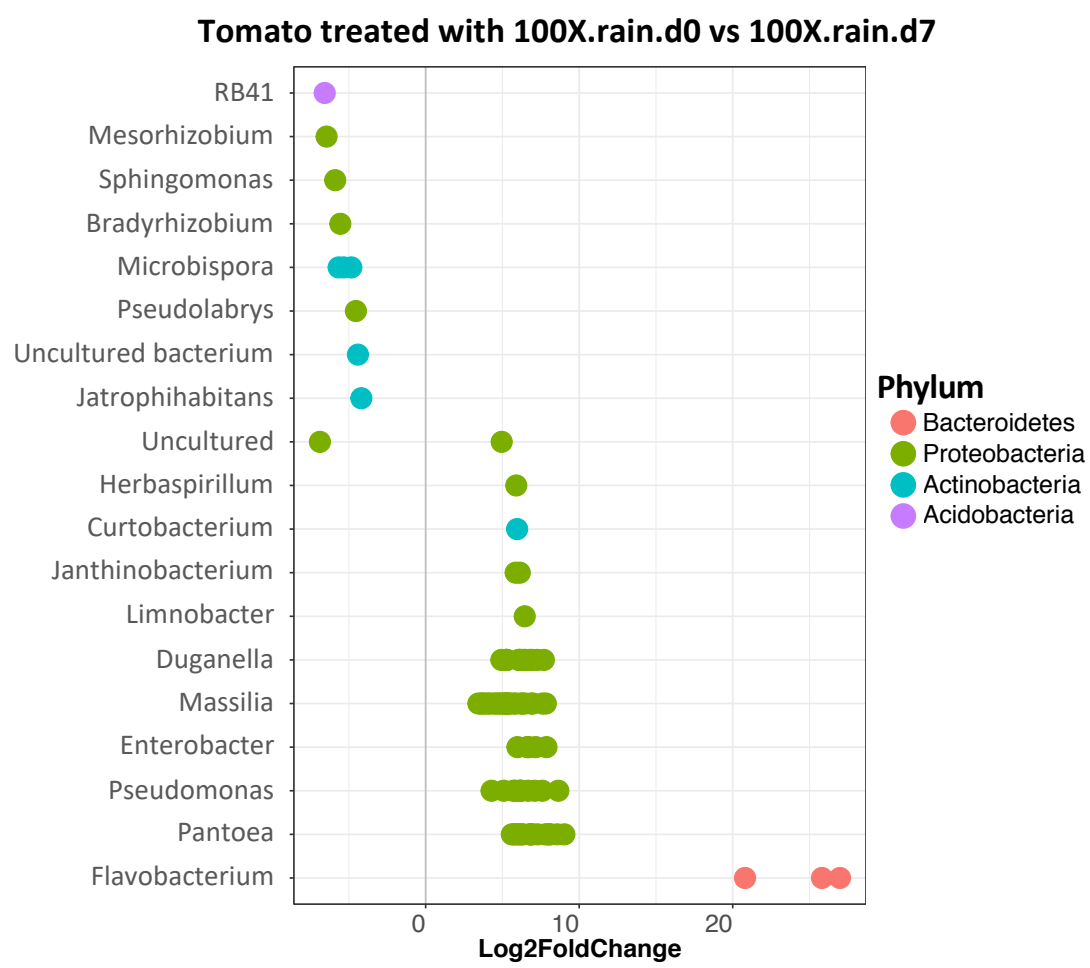

B

Rain vs tomato treated with 100X.rain.d7

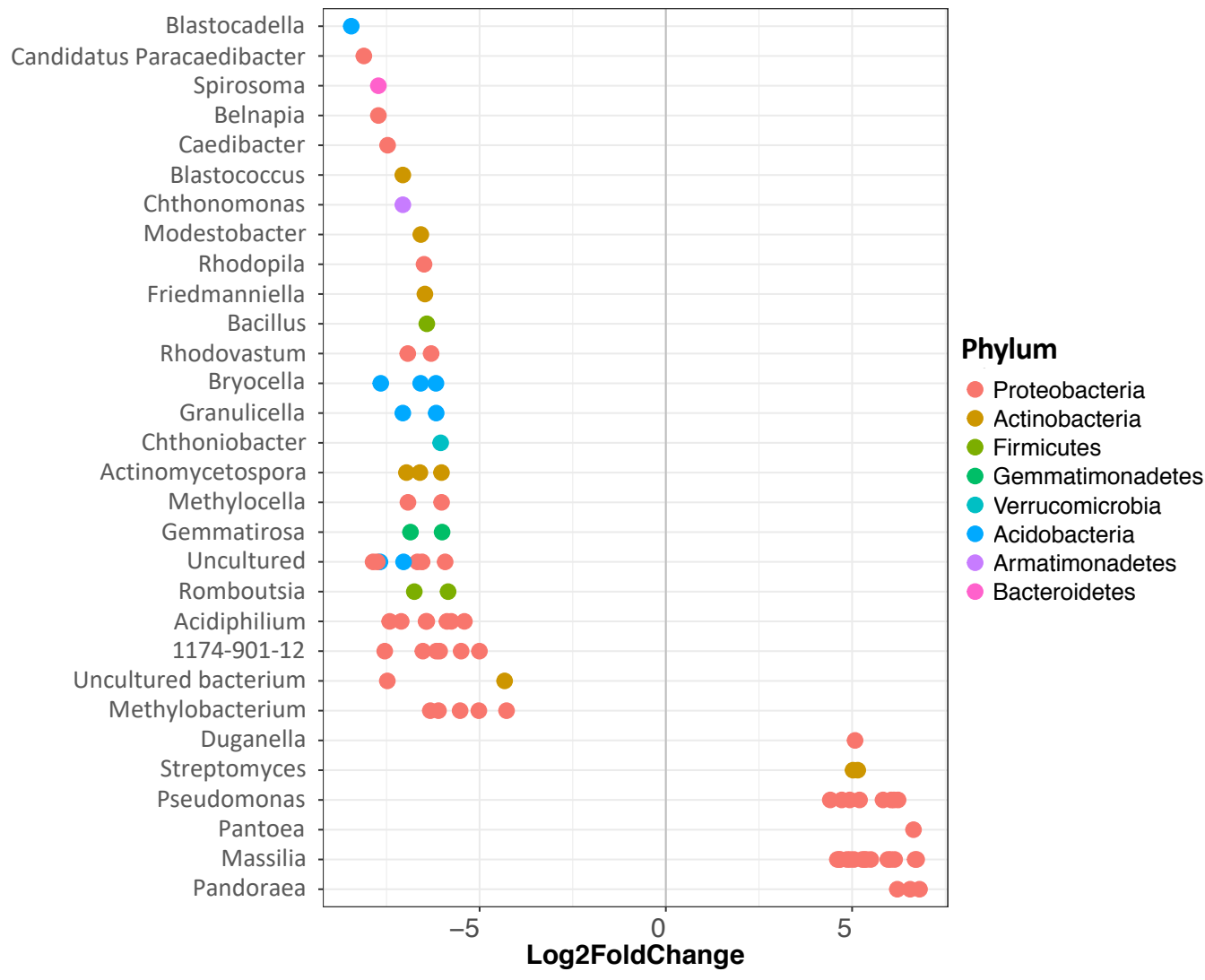

Figure 6: Differential abundance analysis at the level of Operational Taxonomic Unit (OTU) using DESeq2 (Love et al., 2014). The fold change is shown on the $X$ axis and genera are listed on the $Y$ axis. Each colored dot represents a separate OTU. A) Comparison of phyllosphere microbiota of plants treated with 100X-rain between day-0 and day-7, B) Comparison between rain microbiota and phyllosphere microbiota 7 days after treatment with the respective 100X-rain sample. 
A

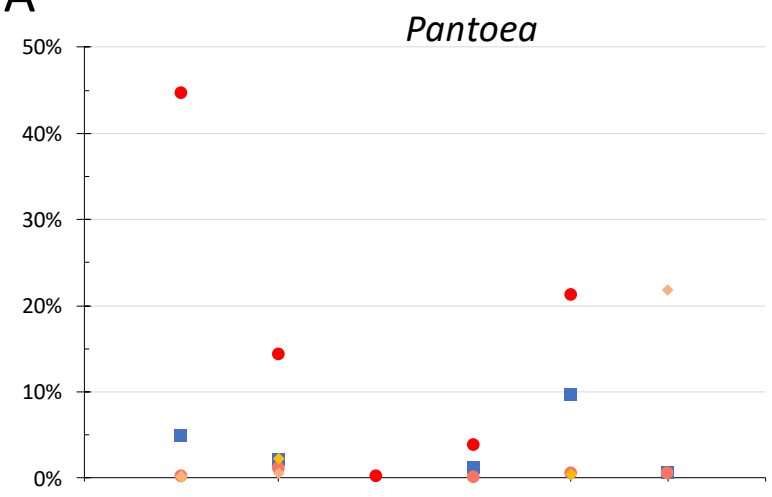

C

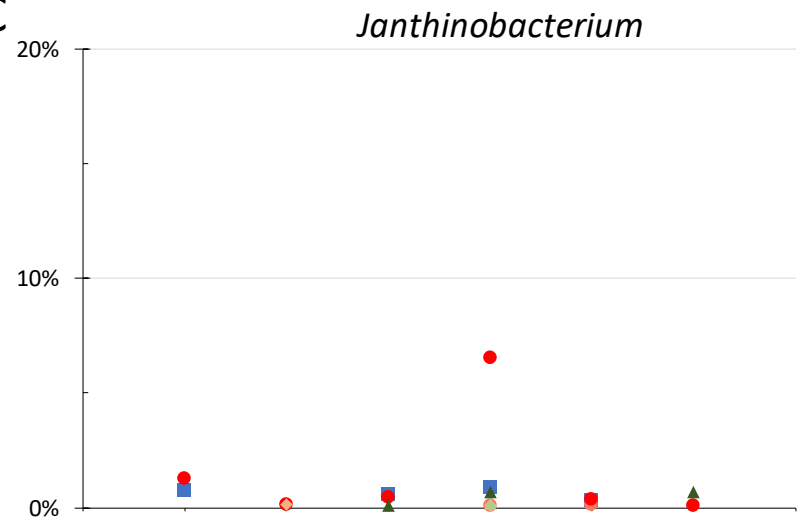

E

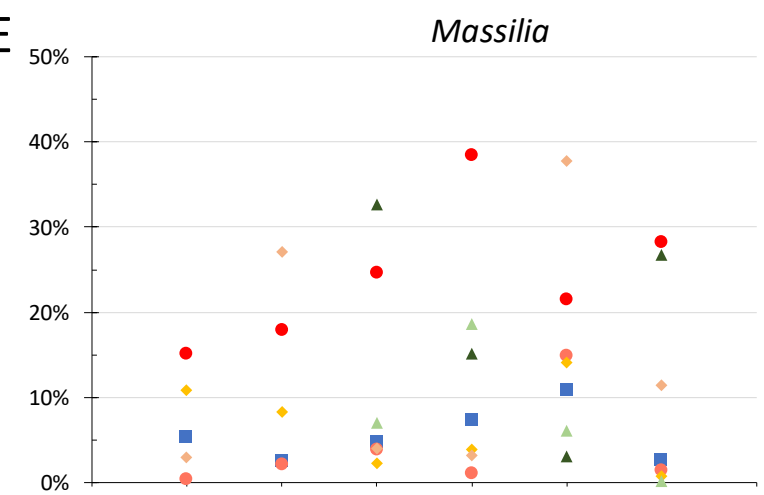

G

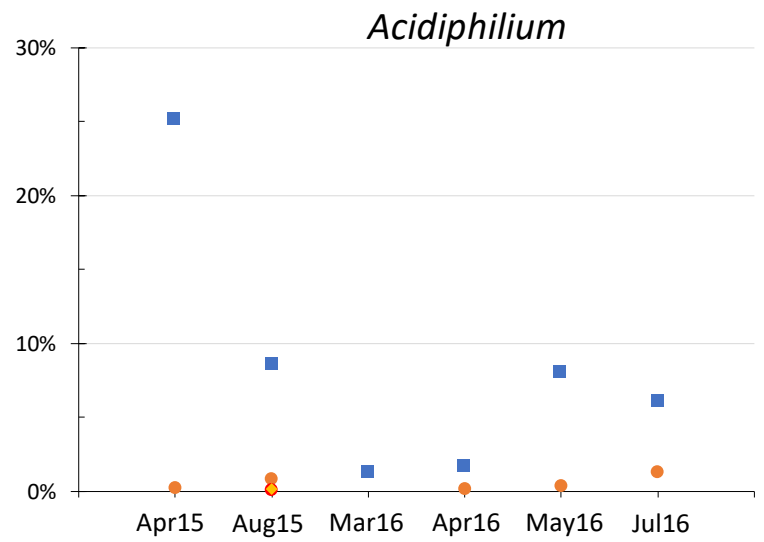

$\mathrm{B}$
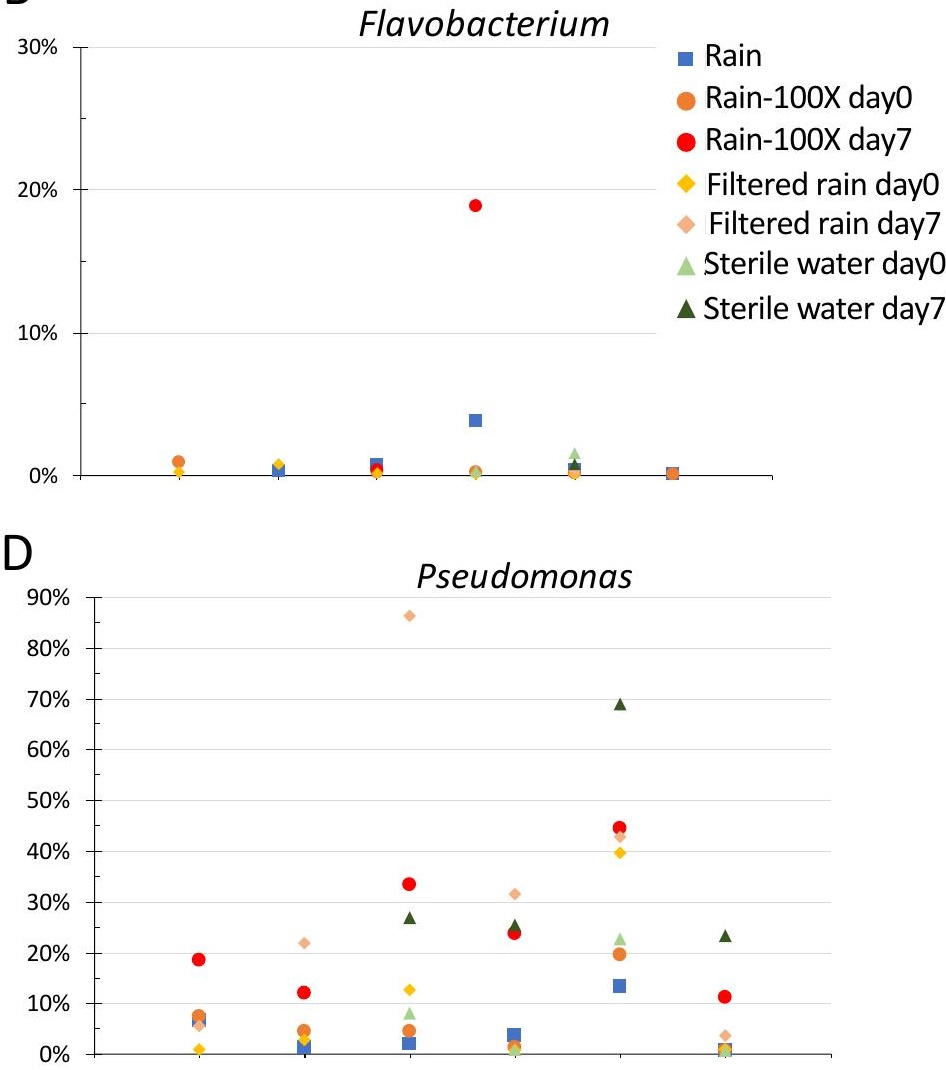

F

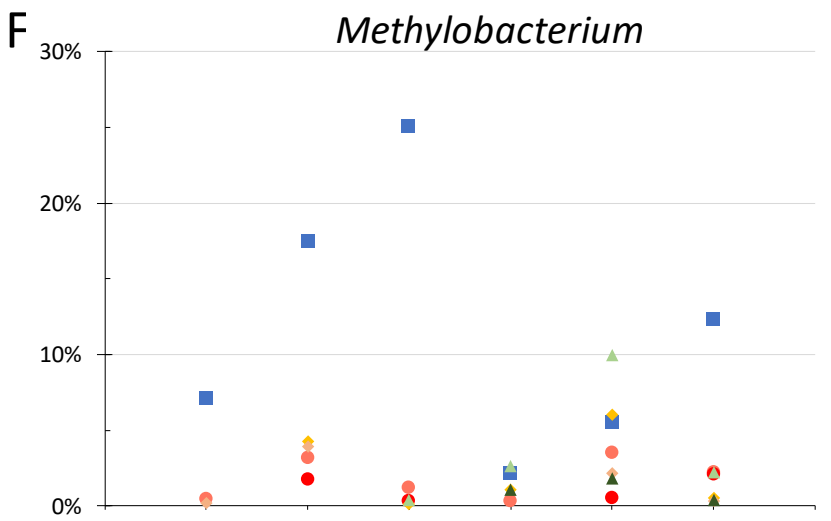

$\mathrm{H}$

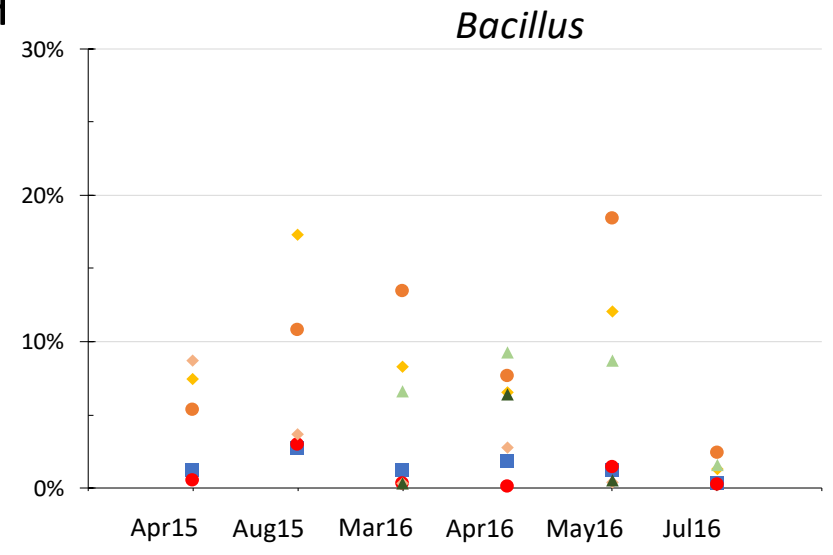

Figure 7. Relative abundance of a representative selection of rain-borne genera that either failed or succeeded in colonizing the tomato phyllosphere. Dates of experiments are listed on the $X$ axis of panels $\mathrm{G}$ and $\mathrm{H}$. Relative abundance is shown on the $\mathrm{Y}$ axis for rain, tomato plants on day- 0 and day- 7 after being treated with 100X-rain, filtered rain, or sterile water (see panel B for figure legend). A) Pantoea, B) Flavobacterium, C) Janthinobacterium, D) Pseudomonas, E) Massilia, F) Methylobacterium, G) Acidiphilium, and H) Bacillus. 


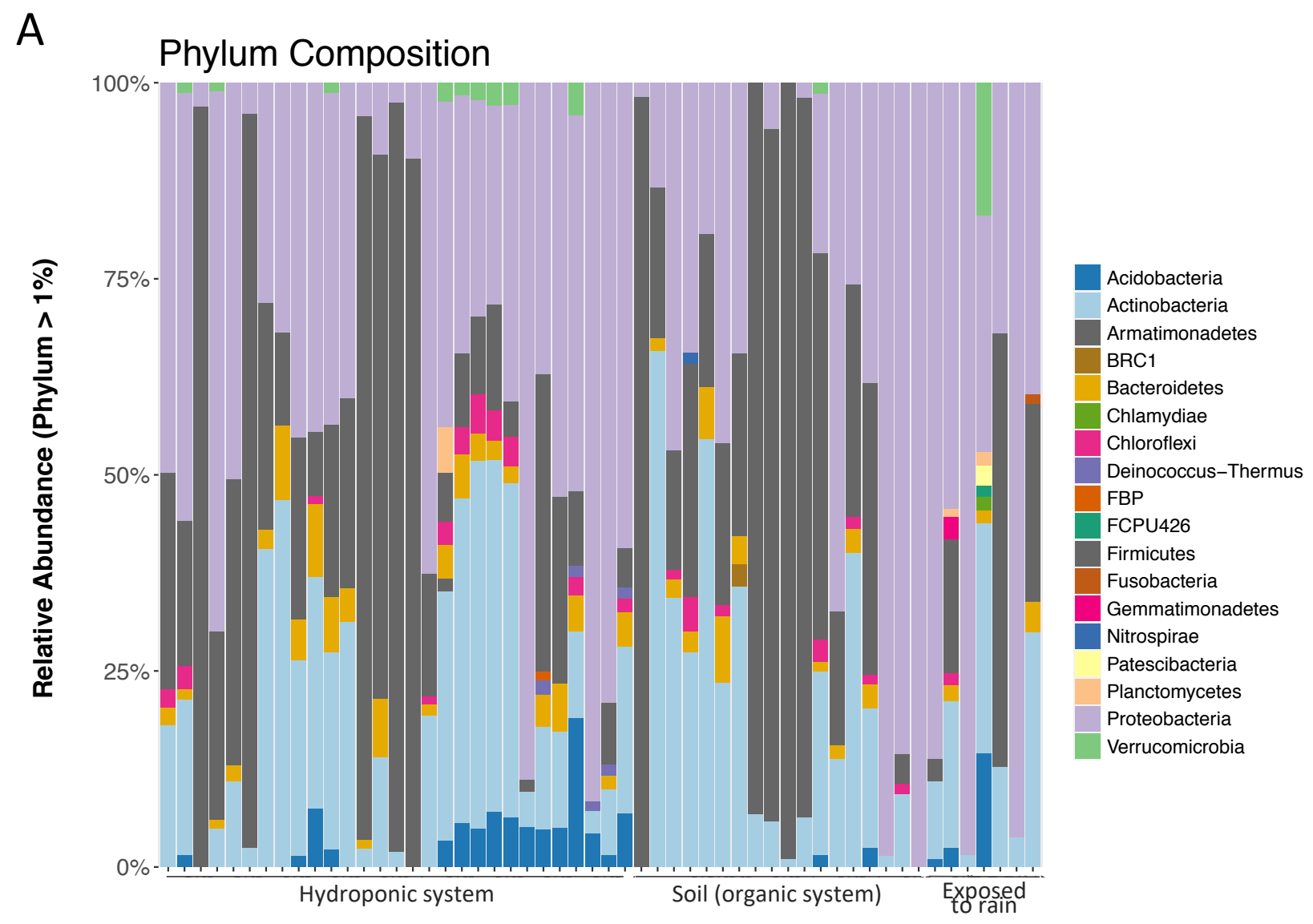

B

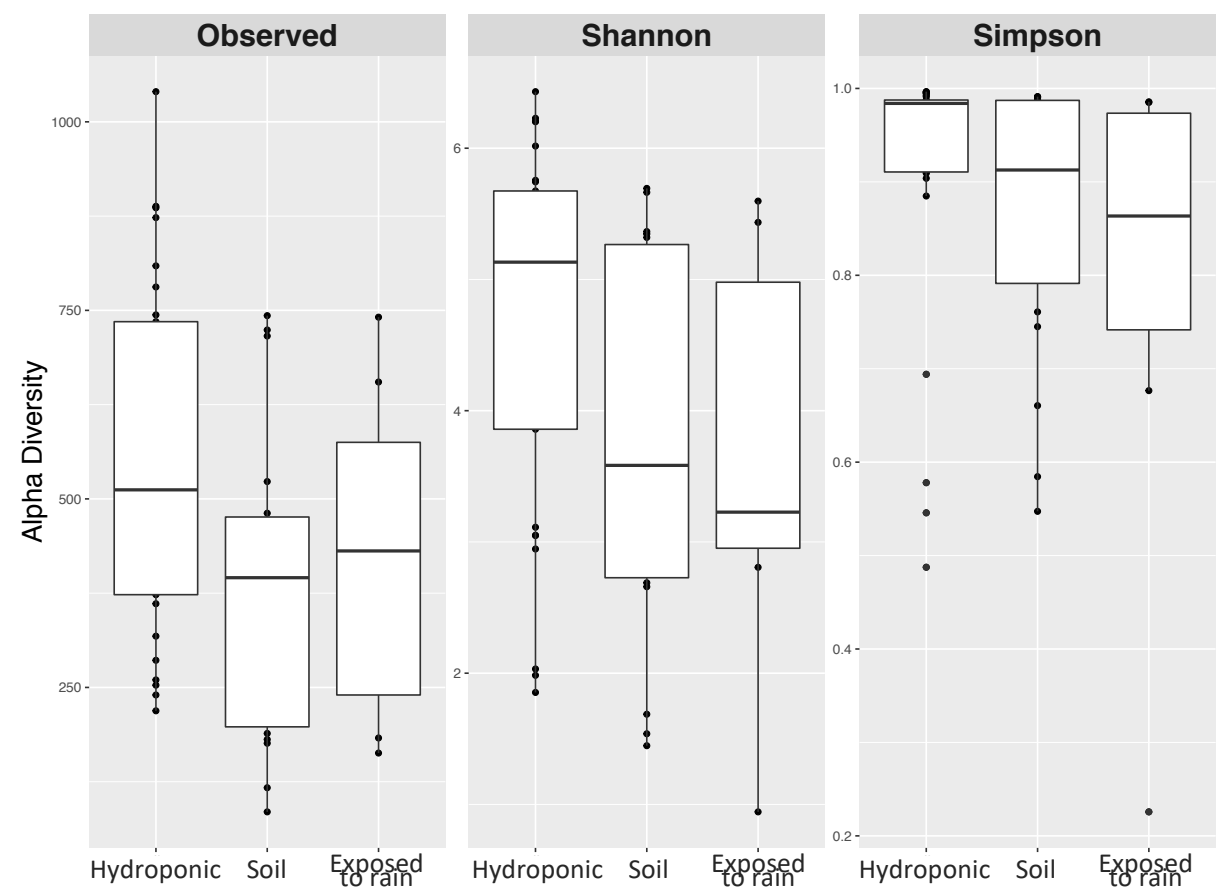

Figure 8. Taxonomic composition and Alpha diversity of phyllosphere microbiota of tomato plants grown hydroponically or in soil (both in a commercial greenhouse) and of tomato plants grown outside (on the roof of the Latham Hall research building). A) Relative abundance at the phylum level (only phyla with a RA above $1 \%$ are shown), B) Alpha diversity (observed OTUs, Shannon diversity index, and Simpson diversity index). 

available under aCC-BY-ND 4.0 International license.

A Tomato plants grown hydroponically vs plants grown outside

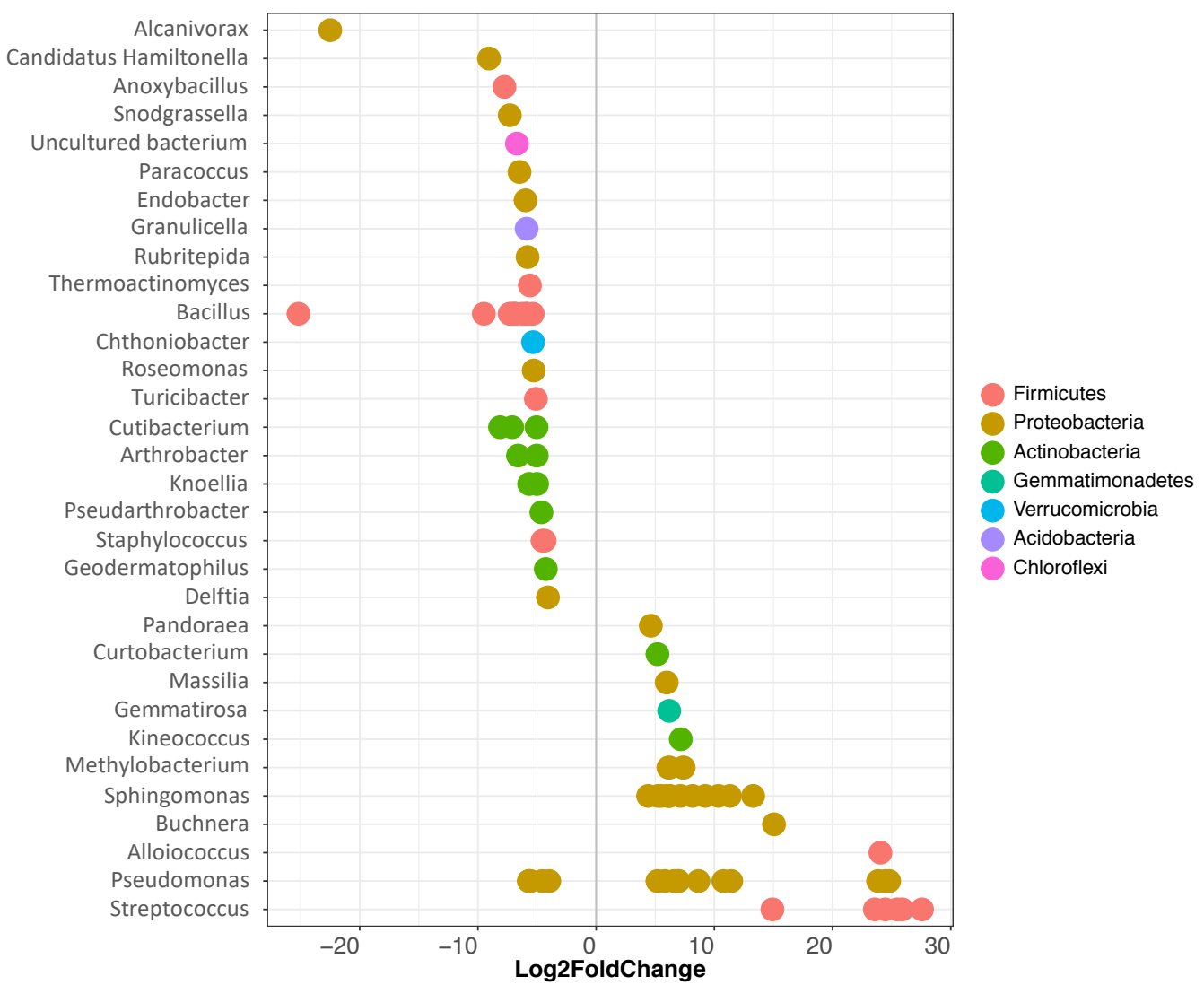

B

Tomato plants grown in soil vs plants grown outside

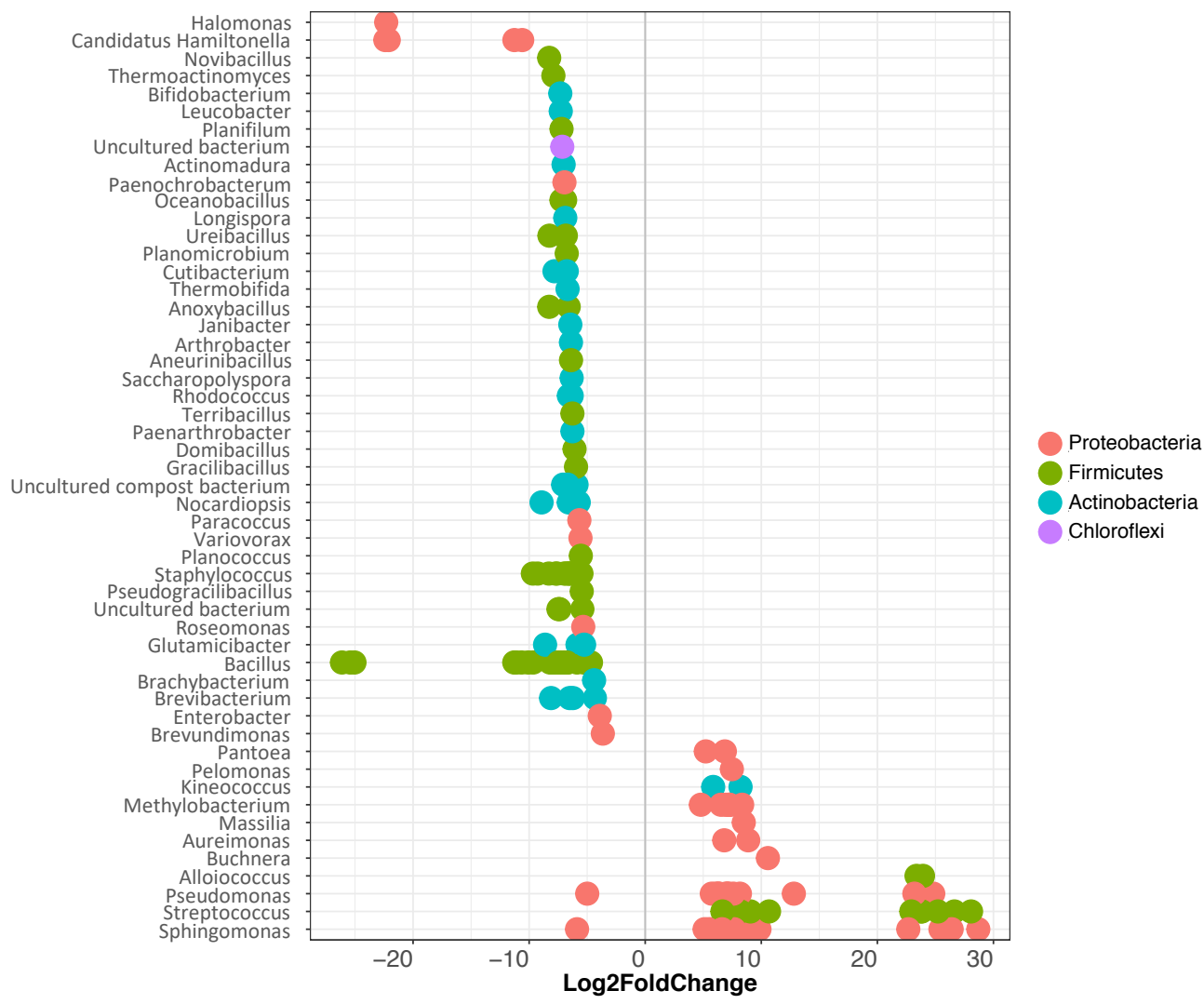

Figure 9. Differential abundance analysis at the level of Operational Taxonomic Unit (OTU) using DESeq2 (Love et al., 2014). The fold change is shown on the $X$ axis and genera are listed on the $Y$ axis. Each colored dot represents a separate OTU. A) Plants grown hydroponically in a greenhouse compared with plants grown on the roof of the Latham Hall research building, B) Plants grown in soil in a greenhouse compared with plants grown on the roof of the Latham Hall research building. 


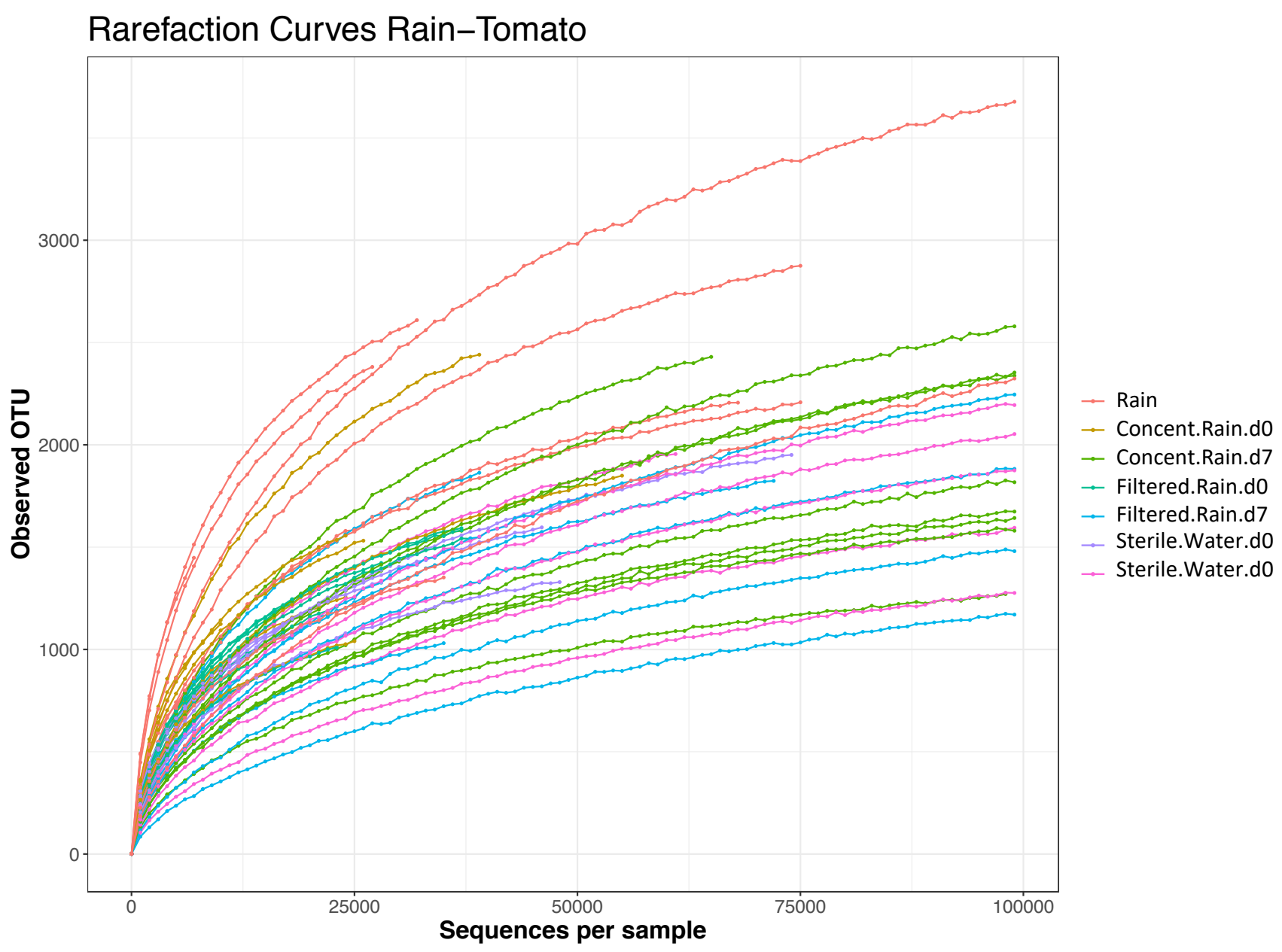

Supplementary Figure 1. Rarefaction curves of rain microbiota and tomato phyllosphere microbiota treated with 100X-rain, filtered rain, or sterile water on day-0 and day-7. 


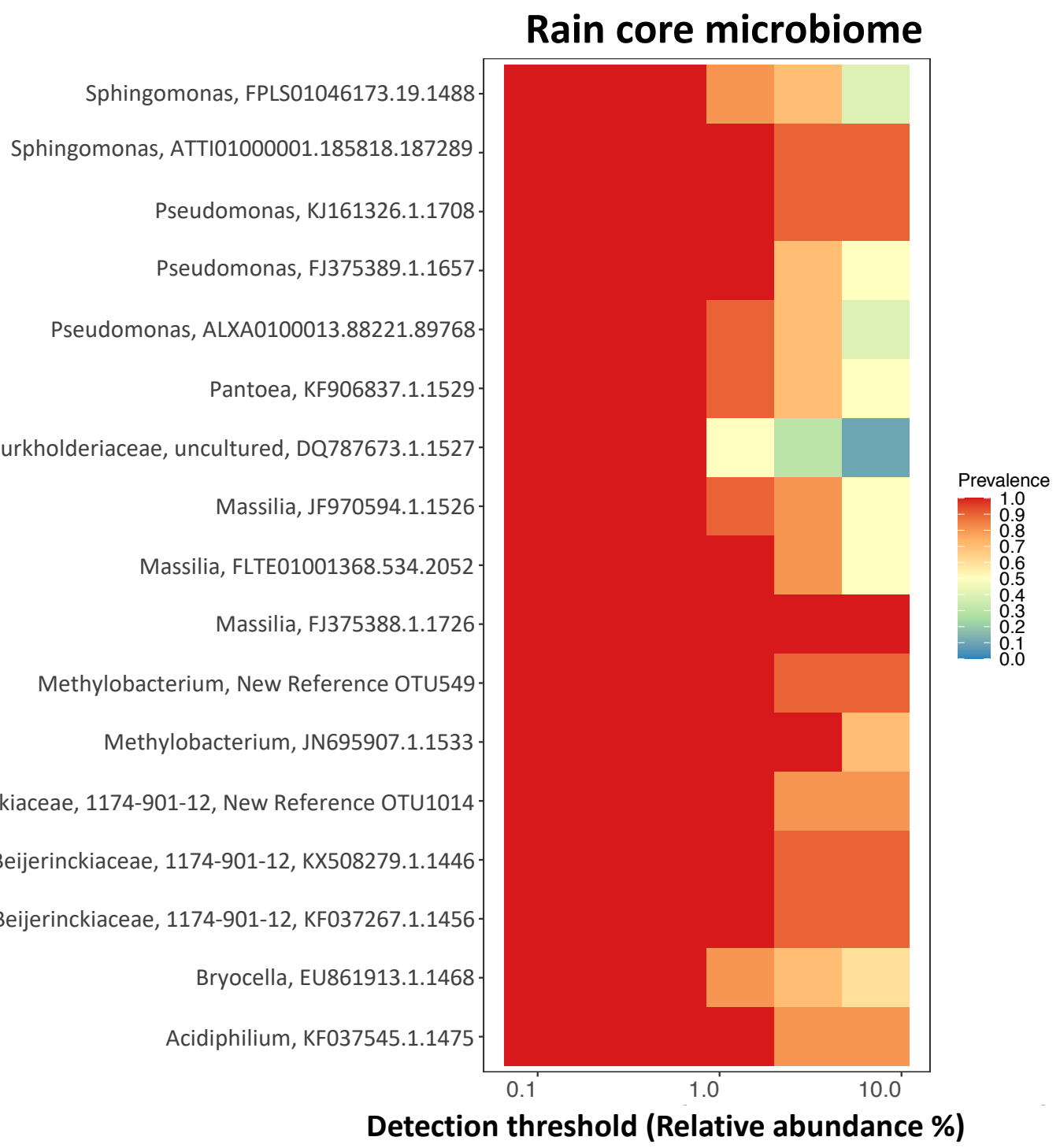

Supplementary Figure 2. The core rain microbiome. Rain-associated OTUs at a detection threshold of $0.1 \%$ and $100 \%$ as a prevalence threshold. 
Rain vs tomato treated with sterile water.do

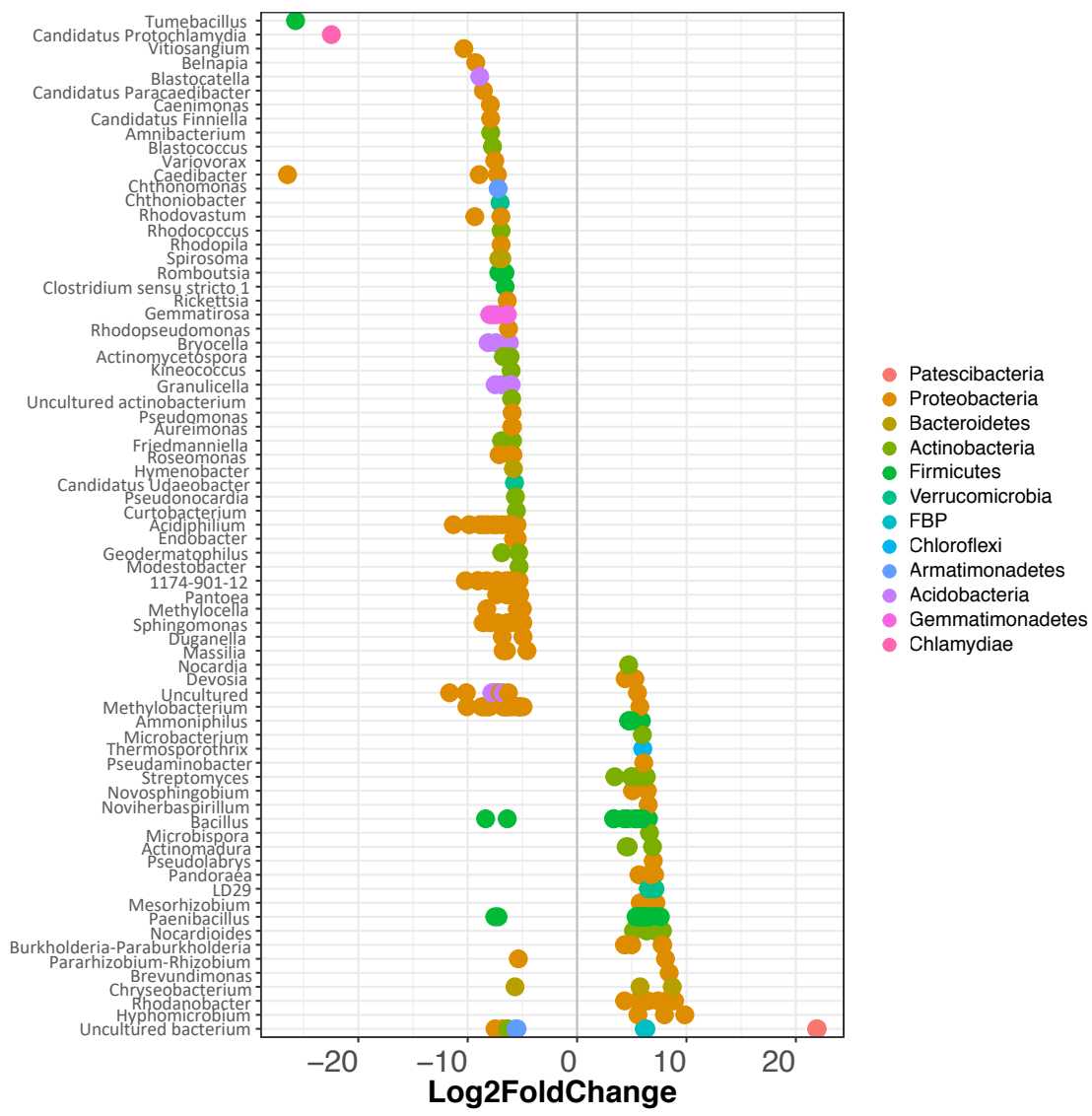

B

Rain vs tomato treated with $100 X$ rain.d0

Candidatus Paracaedibacter

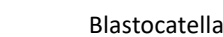

Candidatus Finniella

Rhodopila

Kineococcus

Methylobacterium

Acidiphilium

Sphingomonas

1174-901-12

Rhodanobacter

Novosphingobium

Devosia

Bacillus

Ellin6055

Ammoniphilus

Pseudaminobacter

Pseudolabrys

Streptomyces

Mesorhizobium

Solimonas

Nocardia

Paenibacillus

Microbispora

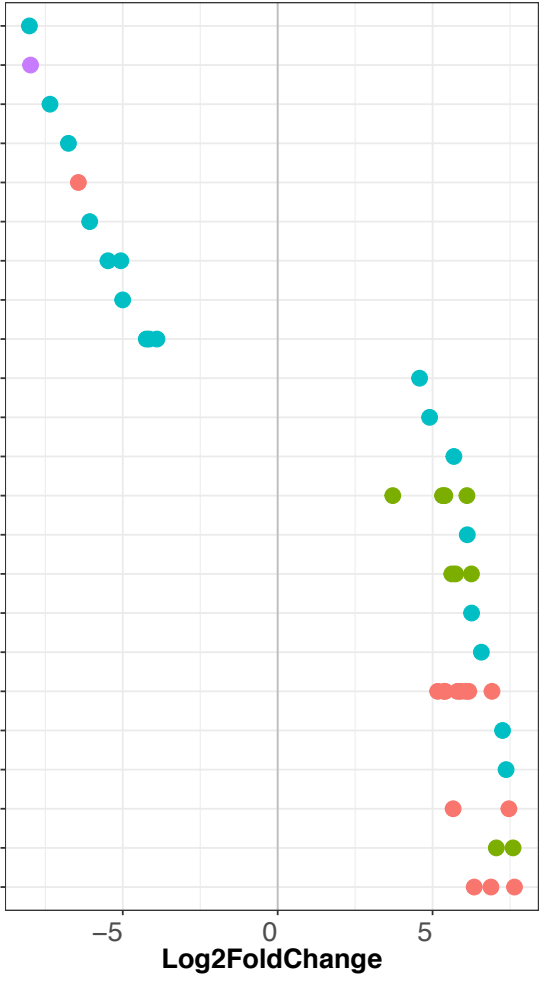

Actinobacteria

- Firmicutes

- Proteobacteria

Acidobacteria

Supplementary Figure 3. Differential abundance analysis at the OTU level usingDESeq2 (Love et al., 2014). A) Comparison of rain microbiota with the tomato phyllosphere treated with sterile water at day- 0 during the same experiment, $B$ ) Comparison of rain microbiota with the tomato phyllosphere microbiota on the day there were treated with 100X-rain (day-0). 